\title{
Studies on the fast ion synergistic effects in JET high performance pulses
}

\author{
K.K. Kirov ${ }^{1}$, Yu. Baranov' ${ }^{1}$, I.S. Carvalho ${ }^{2}$, C.D. Challis ${ }^{1}$, J. Eriksson ${ }^{3}$, D. Frigione ${ }^{4}$, L. Garzotti ${ }^{1}$, \\ J. Graves ${ }^{5}$, P. Jacquet ${ }^{1}$, D.L. Keeling ${ }^{1}$, E. Lerche ${ }^{6}$, P.J. Lomas ${ }^{1}$, C. Lowry ${ }^{7}$, M. Mantsinen ${ }^{8,9}$, \\ F. Rimini ${ }^{1}$ and JET Contributors ${ }^{*}$
}

EUROfusion Consortium, JET, Culham Science Centre, Abingdon, OX14 3DB, UK.

${ }^{1}$ CCFE Fusion Association, Culham Science Centre, Abingdon, United Kingdom

${ }^{2}$ Instituto de Plasmas e Fusão Nuclear, Instituto Superior Técnico, Universidade de Lisboa, 1049-001 Lisboa, Portugal

${ }^{3}$ Department of Physics and Astronomy, Uppsala University, SE-75120 Uppsala, Sweden

${ }^{4}$ Unità Tecnica Fusione - ENEA C. R. Frascati - via E. Fermi 45, 00044 Frascati (Roma), Italy

${ }^{5}$ Ecole Polytechnique Fédérale de Lausanne (EPFL), Swiss Plasma Center (SPC), CH-1015 Lausanne, Switzerland

${ }^{6}$ Lab. for Plasma Phys. KMS-ERM Renaissancelaan 30 Avenue de la Renaissance B-1000, Brussels, Belgium

${ }^{7}$ European Commission, B-1049 Brussels, Belgium

${ }^{8}$ Barcelona Supercomputing Center, Barcelona, Spain

${ }^{9}$ ICREA, Pg. Lluís Companys 23, 08010 Barcelona, Spain.

\begin{abstract}
Fast ion synergistic effects were studied by predictive modelling of JET best performing pulses for various levels of Neutral Beam (NB) and Radio Frequency (RF) power. Calculated DD neutron yields were analysed with the intention of separating the impact of sheer synergistic effects due to changes in fast ion (FI) distribution function (DF) from supplementary effects accompanying the application of RF power, namely changes in $T_{e}$ and $T_{i}$. A novel approach in analysing the efficiency of fast ions in fusion reactions based on evaluation of the cumulative reaction rates is outlined and used in the study. Conclusions on the impact of fast ion synergistic effects on fusion performance are based on comparisons of Beam-Target (BT) and thermal (Th) DD reaction rates. It was found that changes in auxiliary heating power, NB and RF, by $4 \mathrm{MW}$ will affect DD fusion performance and neutron rates significantly. Simulations of the best performing JET pulses show that for $\mathrm{H}$ minority heating scheme with available RF power the impact of sheer synergistic effects is somewhat lesser than the supplementary effects related to changes in $\mathrm{T}_{e}$ and $\mathrm{T}_{\mathrm{i}}$. In conditions of much higher RF power the modification in fast ion distribution function and the impact of the fast ions on DD fusion becomes significant. The impact of the RF and NB power on the BT reactivities was found to be of similar order; however, the NB power has greater impact on reaction rates due to its larger effect on fast ion density.
\end{abstract}

\section{Introduction}

One of the key requirements for achieving high fusion performance in today's tokamaks is injecting high Neutral Beam (NB) power. The beam particles injected with initial energy in the region of 80$120 \mathrm{keV}$ create high-energy fast ion populations in the hot plasma core. Adding Radio Frequency (RF) power leads to interaction between wave electric field and NB ions, providing the wave particle resonance condition is satisfied, and this process further modifies the fast ion distribution function (FI DF). This phenomenon is commonly referred to as "synergistic effects" and it has been a subject of intensive studies as reported in [1-7] and more recently in [8-11]. Better understanding of the

\footnotetext{
* See the author list of "X. Litaudon et al 2017 Nucl. Fusion 57 102001"
} 
physics involved in Fast lon (FI) synergistic effects is important for dealing with two essential issues in present fusion research: (i) improving the fusion performance measured by the neutron rate in stable plasma conditions and (ii) mitigating the negative effect of the interactions between highly energetic particles and various MHD modes.

The need for comprehensive investigation of the interactions between NB fast ions and RF waves in JET has been addressed in a series of recent publications [8-11]. They focus on better understanding of the synergistic effects in view of achieving high fusion performance. Simulations with JETTO/PION codes of JET's best performing hybrid pulses have been carried out [11] and important conclusions have been made regarding RF minority schemes, resonance position and minority concentrations. Deuterium - Tritium (DT) mixture plasmas have been studied extensively in early JET [12-18] and TFTR [18-21] DT campaigns and it has been highlighted that synergetic effects have a key role in achieving high fusion performance.

Fast ion synergistic effects will certainly have implications for JET with the forthcoming DT campaign [22] and future ITER operations [23, 24]. A considerable effort in modelling and optimisation of JET DT neutron yield was carried out in parallel to the recent JET experimental campaign [25]. The main focus of this task was on predictive transport modelling in view of extrapolating from present Deuterium plasma conditions to a DT mixture. In addition to the transport studies it has been recognised [25] that synergistic effects of the NB fast ions will have a considerable contribution to the expected DT fusion performance. Indeed, the maximum of the DT cross-section in the centre-ofmass energy frame is about $64 \mathrm{keV}$, which number translates for $D$ beams energy into cold $T$ target of about $107 \mathrm{keV}$ and for $T$ beam energy into cold $D$ target of about $160 \mathrm{keV}$. JET's NB full energy component is of the order of $80-120 \mathrm{keV}$ for $\mathrm{D}$ beams, i.e. near the maximum of DT cross-section. The full energy component for $\mathrm{T}$ beams is similar, but due to the different form of the cross-section curve for T beam into DT plasma is below energy required for maximum reactivity. This suggests that fine tuning of the fast ion distribution functions for resonant DT reactions can help maximise the fusion yield. Synergistic effects play an important role in this optimisation of the fusion performance and the studies reported here address this issue and contribute towards better understanding of the physics involved.

Neutron rate is a commonly used measure of the fusion performance of neutron producing nuclear reactions. Principal contributions are Beam-Target (BT) and thermal rates. The former is due to collisions of beam fast ions with thermal plasma, while the thermal rates only account for nuclear reactions between bulk thermal ions. Regarding BT neutrons, in this study we focus on two main issues related to fast ion synergistic effects. First, to assess the contribution of sheer synergistic effects resulting from the application of RF power in NB heated plasma. The term "sheer synergistic effect" is used here to refer to the effects due to modification of the fast ion distribution function whereas the term "supplementary effects" refers to changes in electron and ion temperatures, $T_{e}$ and $T_{i}$, from NB and RF heating. Both, $T_{e}$ and $T_{i}$, depend strongly on the applied RF and NB power and separating them from contributions due to RF accelerated fast ion in the analysis of the fusion performance requires closer look at the fast ion distribution function. RF power for instance, changes the electron temperature, $T_{e}$, which will affect the fast ion slowing down and hence will affect the BT neutron rate. Changes to ion temperature, $T_{i}$, on the other hand will strongly affect the thermal nuclear reactions and to some extent BT reactions. For this reason, BT and thermal rates are studied separately in this paper. 
An additional objective of this study is to investigate how different power levels from NB and RF affect the fusion performance. Detailed examination of the fast ion distribution function and DD reaction rates in D plasmas heated with D NB and RF tuned to fundamental H minority frequency, has been carried out to asses which of the two auxiliary heating systems at JET, NB or RF, has a greater impact on the fusion performance. The aim here is to investigate the operational conditions and plasma parameters at which DD neutron yield or equally the DD fusion reaction rates can be maximised. Maximising the input NB and RF power is an obvious choice, but they impact differently on fast ion distribution function as it is shown in this paper. NB power tend to act directly on the fast ion distribution function in the beam energy region, $40-120 \mathrm{keV}$ also called plateau in FI DF here, while RF power in addition to this can pull a high energy tail in the distribution function. The plateau and the tail of FI DF have a different impact on the DD fusion performance and studying this is one of the main subjects of this paper.

Experiments from high power JET campaign in 2016 are discussed and analysed here. Two complementary operational scenarios were developed at JET as main candidates for sustained high DT fusion power [26]: baseline with normalised beta, $\beta_{N} \sim 1.8$, and hybrid with $\beta_{N} \sim 2-3$. The baseline scenario development [27] concentrates mainly on pushing the operation towards the high current and field limits with a relaxed current profile, whereas the hybrid experiments focus mainly on the advantages of operating at high $\beta_{N}$ with a shaped current profile and central safety factor above 1 . Best performing pulses from both scenarios were used in the study. Fully predictive modelling was carried out and reported in [25]. In addition, here power scans were used to investigate the dependence of the reaction rates on the input power. This original approach ensures selfconsistency of the simulations and it provides extra robustness and vigour to the conclusions. Analysing the data by means of predictive modelling, as it was done here, allows for scans in the parametric space as well as separation of various contributions to the investigated neutron rates. An alternative method would be to analyse the database of JET pulses. In these studies, however, this is not helpful in finding underlying parametric dependencies. One of the disadvantages of this approach is that the number of the pulses required for a basic scan in parametric space increases significantly with the number of parameters and values to be scanned. In addition, the fact that many of the input parameters are highly correlated complicates the analysis considerably. For instance, RF power will change electron and ion temperature hence by using database analysis method there will be no clear indication of whether $T_{i}$ and $T_{e}$ changes or synergistic effects due to application of RF power have the greatest impact on the neutron rates. As an example to illustrate this, in figure 1 shown are the neutron yield versus diamagnetic energy in all high-power baseline pulses at JET in 2016 campaign. Color-coded points depict the dependencies on the applied RF power in a) and central electron temperature in b). Clearly pulses with high diamagnetic energy need higher RF power in order to achieve higher neutron rate; however, similar dependence is seen with regard to electron temperature as well. Obviously, this kind of analysis is incapable of distinguishing between impact of the synergistic and supplementary effects on the fusion performance. Thus, in this paper the preferred method of analysis is by predictive modelling of the fusion performance by scanning the input RF and NB power. 


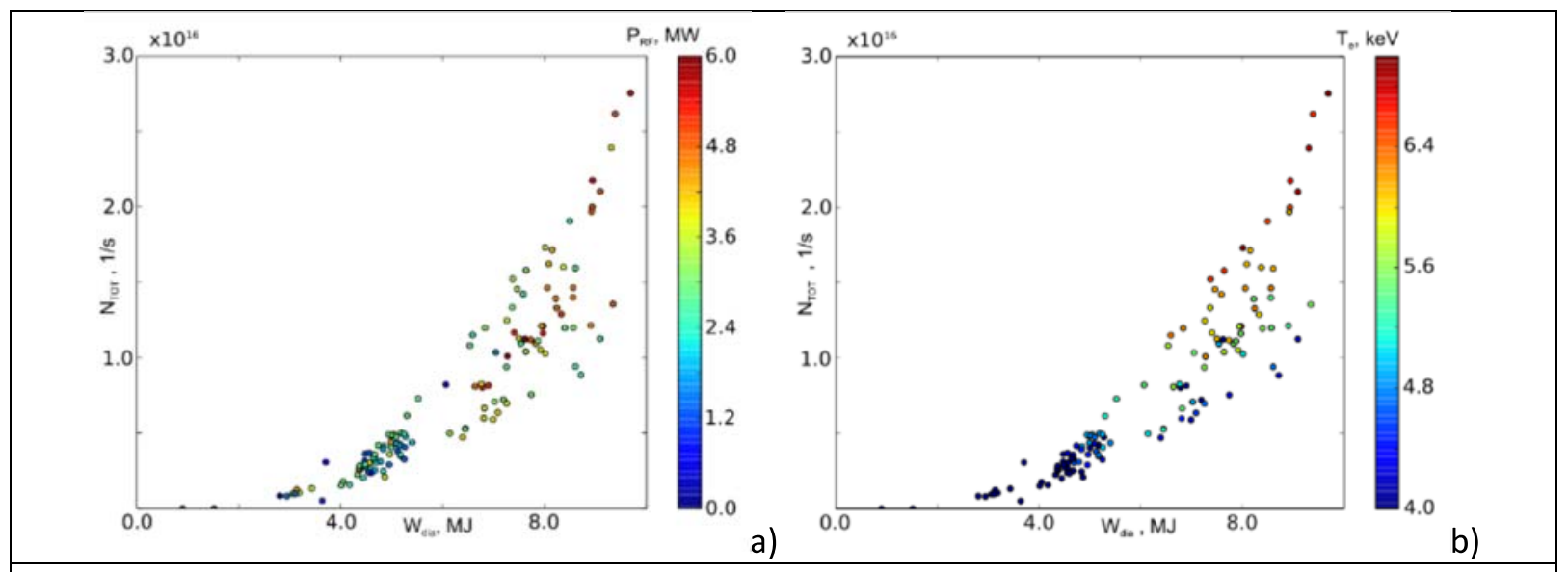

Figure 1: Neutron yield, $\mathrm{N}_{\text {TOT, }}$, versus diamagnetic plasma energy, $\mathrm{W}_{\text {dia }}$, for baseline database with colour-coded RF power $P_{R F}$ in a) and electron temperature $T_{e}$ in $b$ ).

Simulations of the best performing JET pulses show that for $\mathrm{H}$ minority heating scheme with available RF power the impact of sheer synergistic effects is somewhat lesser than the supplementary effects with varying the heating power. In conditions of much higher RF power the modification in FI DF and the impact of the fast ions on DD fusion becomes significant. The impact of the RF and NB power on the BT reactivities was found to be of similar order; however, the NB power has greater impact on reaction rates due to its larger effect on fast ion density. This paper is organised as follows: Section 2 gives details on the numerical codes used. In section 3 important diagnostics on which this study heavily relies are discussed together with the high-performing baseline and hybrid pulses. Detailed physical picture of the fast ion distribution function modifications by NB energy and RF electric field are provided in section 4. Results are presented in section 5 where the findings of the predictive modelling and the power scans are highlighted. Section 6 is dedicated to conclusions and discussions.

\section{Modelling tools used in the study}

Routine predictive transport modelling at JET is usually performed with the JETTO code [28] coupled to PION/PENCIL package for computing NB and RF power absorptions taking into account the synergistic effects. Various transport models can be used in JETTO based on first principles physics or empirical scaling. A distinctive semi-empirical model which gives reasonable agreement with a large proportion of JET experimental data is the Bohm-gyroBohm model [29], which uses combination of Bohm and gyro-Bohm terms in the heat diffusivity expression.

The PION code [30] is used in JETTO for ICRH minority and harmonic heating utilising its main advantage of being computationally fast thus compatible with integrated modelling. The code interfaces with the existing PENCIL NB deposition code [31], [32] and accounts for NB and RF synergy effects [33] thus providing flux-surface averaged fast ion distribution function and RF power deposition self-consistently. PION cannot be used with mode conversion schemes or cases with large minority fraction, where mode conversion becomes significant. For the case considered here, however, the minorities concentrations are very small, lower than necessary for any considerable mode conversion to take place, so the impact of this is negligible. The orbit effects are treated in a simplified way in POIN; however, due to its simplicity, they are neglected in PENCIL thus important physics of the fast ion distribution and confinement is missing in this treatment. 
In addition to the predictive JETTO modelling the TRANSP [34-37] code was used to provide fast ion distribution functions and BT reactivities as well. The NUBEAM code [38] is a computationally comprehensive Monte-Carlo code for NB injection in tokamaks. The code follows the fast ion trajectories and takes into account orbit effects in fast ion distribution calculations which is its main advantage over PENCIL.

The principal RF wave solver for TRANSP is the TORIC code [39]. In TRANSP, TORIC is coupled to a Fokker-Planck solver, FPP [40], which adds bounce averaging treatment. To study the ion cyclotron (IC) resonance of the heated ions, Monte Carlo quasi liner RF kick operator [53] was implemented in NUBEAM and used in the study. The RF wave solver in TRANSP, TORIC, provides information about $\mathrm{RF}$ electric field components and perpendicular wave vector for each toroidal mode. RF resonance condition for a given harmonic is then used to calculate the magnetic moment and energy of the particles satisfying the resonant condition. Assuming that the resonant ions lose their phase information with RF wave by collisions and wave stochasticity before they re-enter the resonance layer a random walk model can be used to reproduce the stochastic nature of RF heating in magnetic moment space. Every time fast ion passes through resonance layer it receives a kick in magnetic moment space. The magnitude of the kick is derived from the quasi-linear theory, while the stochastic nature is reproduced by means of Monte Carlo random number for the phase of the gyroorbit. Details of the implementation of RF kick operator in NUBEAM code and results of various benchmarking tests are provided in [53]. At present there is no feedback from NUBEAM's fast ion distribution function to TORIC.

\section{Experimental setup}

\subsection{Essential diagnostics}

Experimental data from standard JET diagnostics and recommended signals were used in the study. Density profiles and electron temperature profiles were taken from the High Resolution Thomson Scattering diagnostics, HRTS, and/or Llght Detection And Ranging, LIDAR, measurements [41]. Electron temperature from ECE radiometer [42] was also included in the analysis. Radiated power was measured by the bolometric diagnostics, while $Z_{\text {eff }}$ was assessed by means of Bremsstrahlung measurements from visible spectroscopy. Neutron production rates were taken from the available neutron yield monitors.

After the change of JET wall from C to Be and W metallic ITER Like Wall (ILW) traditional chargeexchange spectroscopy for $\mathrm{T}_{i}$ measurements, heavily relying on CVI spectra analysis, has become considerably more difficult. A combination of diagnostics was used to deduce $T_{i}$ for the investigated pulses: X-ray crystal spectroscopy (XCS), Charge eXchange Recombination Spectroscopy (CXRS) and neutron spectrometer.

\subsection{JET neutron spectrometer TOFOR}

Data from JET neutron Time-Of-Flight spectrometer (TOFOR) were exclusively used in the neutron spectra analysis. The TOFOR diagnostic is described in detail in $[43,44]$. It has a vertical line of sight through the plasma core and perpendicular to the magnetic field covering the region between $2.74 \mathrm{~m}<R_{\mathrm{maj}}<3.02 \mathrm{~m}$. TOFOR consists of two sets of plastic scintillator detectors. First is placed in the collimated flux of neutrons from the plasma and the second is placed 1.2 meters away at an angle of 30 degrees to the beam direction. A fraction of the incoming neutrons scatter in the first detector and then some of them are detected by the second one. The time of each scattering event is 
recorded and from the two arrays of scattering times a time-of-flight (TOF) spectrum is constructed. The energy of incoming neutrons is determined by the TOF related to the measured distance between the two detectors. DD neutrons, which typically have energies of about $2.5 \mathrm{MeV}$, give rise to flight times around 65 ns. The full response function of TOFOR has been calculated with MonteCarlo methods [45]. For the cases simulated and discussed here TOFOR time-resolution is a limiting factor; in order to obtain data with reasonable confidence one has to integrate over $0.5 \mathrm{~s}$ around the time of interest.

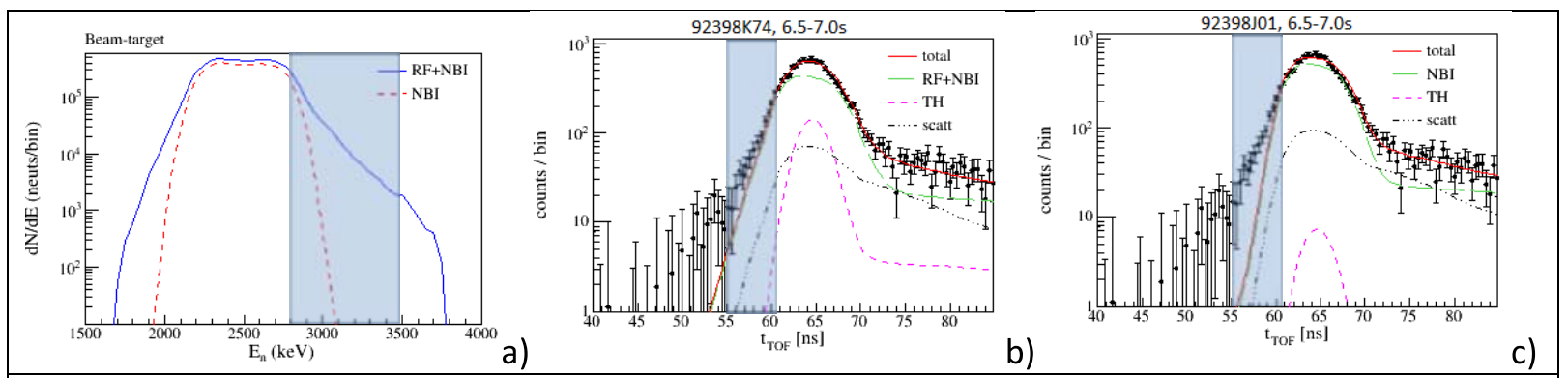

Figure 2: a) Beam-target DD 2.45MeV neutron production spectra showing that RF contributions can be clearly identified for neutron energies $E_{n}<2 \mathrm{MeV}$ and $\left.E_{n}>2.8 \mathrm{MeV} ; b\right)$ TOFOR analysis compared to simulated $\mathrm{t}_{\mathrm{TOF}}$ for hybrid pulse $\# 92398$ with $\mathrm{P}_{\mathrm{RF}}=5 \mathrm{MW}$ showing good agreement between simulations and measurements; c) TOFOR analysis compared to simulated $t_{\text {TOF }}$ for hybrid pulse \#92398 with zero RF power and lower $T_{e}$ and $T_{i}$ showing that the high energy (short time-of-flight, $<61 \mathrm{~ns}$ ) part of the spectrum does not match the TOFOR data for $55 \mathrm{~ns}<\mathrm{t}_{\mathrm{TOF}}<61 \mathrm{~ns}$.

Beam-target neutron spectra, $\mathrm{dN} / \mathrm{dE}$, for $\mathrm{NB}+\mathrm{RF}$ and $\mathrm{NB}$ only cases are shown in an illustrative example in figure 2 a). Significant enhancement of $d N / d E$ by the RF power is expected for lower, $E_{n}<2 \mathrm{MeV}$, and higher energies, $E_{n}>2.8 \mathrm{MeV}$. Monoenergetic beams with energies of $100 \mathrm{keV}$ and $500 \mathrm{keV}$ are expected to create double-humped shaped neutron spectra with high-energy peaks at $E_{n}=2.8 \mathrm{MeV}$ and $E_{n}=3.5 \mathrm{MeV}$ respectively. These estimates of $E_{n}$ correspond to $t_{\text {TOF }}=61 \mathrm{~ns}$ and $t_{\text {TOF }}=55 \mathrm{~ns}$ [24]. This constitutes the basis of detection of fast ions created by RF by means of the TOFOR diagnostic and neutron energy ranges and $t_{T O F}$ time which correspond to these values are highlighted in figure 2. TOFOR data are compared to simulated data assuming $5 \mathrm{MW}$ of RF power figure $2 \mathrm{~b}$ ). In addition, a case without RF power and $10 \%$ lower $T_{e}$ and $T_{i}$ is shown in figure $2 \mathrm{c}$ ). The good match of $t_{\text {TOF }}$ in the range $55 \mathrm{~ns}$ to $61 \mathrm{~ns}$ in figure $2 \mathrm{~b}$ ), which is the band where RF effects are most clearly observed (given in grey in figure 2) suggests the fast ion distribution function from TRANSP run with $5 \mathrm{MW}$ of RF power matches reasonably well the experimental observations. On the other hand, the calculation assuming no RF power, hence no FI DF tail, figure $2 \mathrm{c}$ ), clearly contradicts TOFOR observation for $\mathrm{t}_{\text {TOF }}$ between $55 \mathrm{~ns}$ and $61 \mathrm{~ns}$.

\subsection{JET high performance baseline and hybrid pulses}

The baseline [26] development experiment on JET [27] featured a number of high performing pulses at high plasma current and input power. Pulse \#92436, figure 3 a), had characteristics: $3 M A / 2.8 T$ with line integrated density of $\approx 1.8 \times 10^{20} \mathrm{~m}^{-2}$ (line averaged of about $6.4 \times 10^{19} \mathrm{~m}^{-3}$ ) and central $\mathrm{T}_{\mathrm{e} 0} \approx 7.3 \mathrm{keV}$ and $\mathrm{T}_{\mathrm{i}}$ near the plasma core of about $8-9 \mathrm{keV}$. NB power of about $27.5 \mathrm{MW}$ was applied right at the end of the current ramp up at $7.55 \mathrm{~s}$. ICRH power in dipole at $42.5 \mathrm{MHz}$ was ramped from $8.05 \mathrm{~s}$ and reached its maximum of about 5.2 $\mathrm{MW}$ half a second later for $\mathrm{H}$ minority heating, while the radiated power measured by the bolometric measurements was about $40 \%$ of the total input power. 
Gas dosing during the main heating phase increases from $\approx 6.4 \times 10^{21} \mathrm{el} / \mathrm{s}$ to about $9.4 \times 10^{21} \mathrm{el} / \mathrm{s}$ with H concentration of about $3 \%$ of the total electron density. Small $2 \mathrm{~mm}$ pellets were fired with frequency of about $45 \mathrm{~Hz}$ from $8.5 \mathrm{~s}$ to $13 \mathrm{~s}$ to maintain plasma density and sustain regular ELMs. Pellets are fired only into the periphery of the plasma, so that their impact could be modelled by gas puff only. Type I ELMs with frequency of about $30 \mathrm{~Hz}$ were observed up until $\approx 10.6 \mathrm{~s}$. The pulse featured reasonable confinement enhancement with $H 98 \approx 1.1$, relatively high normalised beta, $\beta_{\mathrm{N}} \approx 2$, and neutron rate up to $2.8 \times 10^{16} \mathrm{~s}^{-1}$. The pulse was modelled in TRANSP and by JETTO from the start of the main heating phase, $7.5 \mathrm{~s}$, up until $9.4 \mathrm{~s}$.

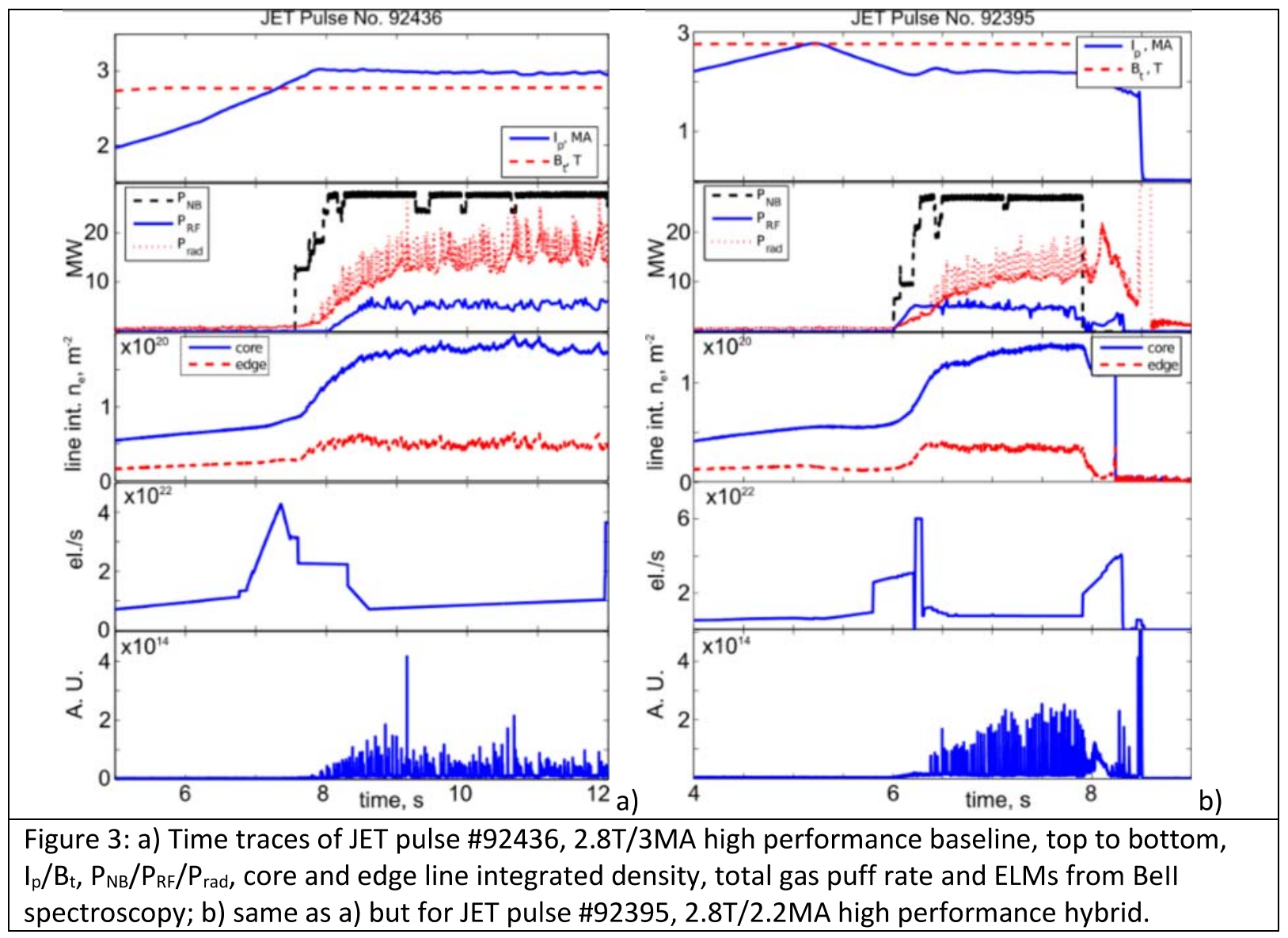

JET pulse \#92935, figure 3 b), was one of the best performing hybrid scenario [26] pulses in the last JET campaign regarding neutron rates. The pulse was carried out as part hybrid scenario development experiment and its main parameters are as follows: $2.2 \mathrm{MA} / 2.75 \mathrm{~T}$, line integrated density $\approx 1.3 \times 10^{20} \mathrm{~m}^{-2}$ (line averaged of about $4.6 \times 10^{19} \mathrm{~m}^{-3}$ ), central $\mathrm{T}_{\mathrm{e} 0} \approx 7.8 \mathrm{keV}$ and $\mathrm{T}_{\mathrm{i}}$ near the core of about $8 \mathrm{keV}$ were achieved by means of $27 \mathrm{MW}$ of NBI power and $5 \mathrm{MW}$ of ICRH in dipole at $42.5 \mathrm{MHz}$ for $\mathrm{H}$ minority heating with steady radiation assessed by bolometric measurements to be of about $30 \%$ of the total input power. Gas dosing during the main heating phase was about $7 \times 10^{21} \mathrm{el} / \mathrm{s}$ maintaining steady ELMs with frequency of about $35 \mathrm{~Hz}$ while the target $\mathrm{H}$ minority concentration was kept of about 1.5\%. Confinement was of the order of H98 1.3 for about $1 \mathrm{~s}$ from the start of the main heating. Normalised beta $\beta_{\mathrm{N}} \approx 2.5$ was sustained throughout the pulse, while neutron rates up to $2.7 \times 10^{16} \mathrm{~s}^{-1}$ were measured, which is one of the highest for ILW hybrid pulses. Large fishbones and $\mathrm{n}=3$ mode were observed at about 7.9s. A main chamber hot spot alarm at $7.9 \mathrm{~s}$ triggered controlled 
termination of the pulse a few hundred milliseconds after that (at about 8.4s). The pulse was modelled in TRANSP and by JETTO from the start of the main heating phase, $6 \mathrm{~s}$, up until 7.8s.

\section{Impact of RF electric field on NB fast ions}

Results presented in this study will be discussed by means of fusion reactivities and reaction rates. For the cross-section, $\sigma$, reactivity, $\sigma . v$, and the averaged reactivity, $\langle\sigma . v\rangle$, standard notations, definitions and units are used. Averaged reactivity by the thermal (Th) ions is noted as $\langle\sigma . v\rangle_{\text {Th, }}$ while for Beam-Target (BT) reactions $\langle\sigma . v\rangle_{\mathrm{B} \text { T }}$ notation is used. In addition, $\mathrm{BT}$ reactivities are assessed by means of formula (7) from Mikkelsen paper [46]. This formula, which here will be referred to as $\langle\sigma . v\rangle_{\text {BT_MEB }}$, is a good approximation for $B T$ reactivities in $D(d, n)^{3} H$ He reactions and beam energies of up to $0.7 \mathrm{MeV}$. It takes into account the temperature of the target plasma but is only valid for monoenergetic beams (MEB). The expression for the full BT reactivity $\langle\sigma . v\rangle_{\text {BT }}$ can be derived in principle after integration of the product of $\langle\sigma . v\rangle_{\text {BT_MEB }} f_{f i}$ over fast ion energies, where

$\mathrm{f}_{\mathrm{fi}}(\mathrm{E})$ is normalised fast ion distribution function, $\int_{0}^{\infty} f_{f i}(E) d E=1$. The latter is related to the total fast ion distribution function, $\mathrm{F}_{\mathrm{fi}}(\mathrm{E})=\mathrm{n}_{\mathrm{fi}} \mathrm{f}_{\mathrm{fi}}(\mathrm{E})$, via the fast ion density, $\mathrm{n}_{\mathrm{fi}}$. In order to assess the contribution of the fast ions to the fusion performance, the product $\langle\sigma . v\rangle_{\text {BT_MEB }} F_{f i}$ as a function of fast ion energy, E, and the cumulative integral of it will be used in the study. The latter is calculated in the interval $[0, E]$ and is also presented as a function of $E$. For infinitely large $E$ the cumulative integral is equal to $n_{\mathrm{fi}}\langle\sigma . v\rangle_{\mathrm{BT}}$, which is also used for consistency checks. By using the product $\langle\sigma . v\rangle_{\text {BT_MEB }} F_{f i}$ and the cumulative integral of it as a function of the energy instead of the full integral, an estimate of how fast ions contribute to BT reactivity can be made. In this way the contribution of high energy fast ions to the fusion performance can be highlighted. For instance, as the synergistic effects will impact on FI DF the change of the value of the cumulative integral for due to changes in RF power will show directly the impact of the sheer synergistic effects. Reaction rate is proportional to the densities of the reactants and averaged reactivity, i.e. $R_{B T}=n_{f i} n_{D}\langle\sigma . v\rangle_{B T}$ and $R_{T h}$ $\left.=n_{D}{ }^{2}<\sigma . v\right\rangle_{T h} / 2$, where $n_{f i}$ and $n_{D}$ are the fast ion and bulk deuterium densities. Volume integral of $\mathrm{R}_{B T}$ and $\mathrm{R}_{T h}$ will provide the total number of neutrons per second, $\mathrm{N}_{T O T}=\mathrm{N}_{B T}+\mathrm{N}_{T h}$, from the plasma. This can be measured with good accuracy over a wide range of energies by the available neutron detectors at JET. The beam-beam (BB) neutron rates are assessed to be at least two orders of magnitude lower than thermal and BT rates, so this contribution is neglected in the analysis.

A number of processes and plasma parameters determine the evolution of the beam particles population in hot dense thermonuclear plasma. A brief account of all the processes involved in fast ions evolution and underlying physics is given here. Once the particles of the propagating neutral beam enter the dense plasma they are ionised and confined in the tokamak's magnetic field. lons with full beam energy, $E_{b}$, usually in the range of about $100 \mathrm{keV}$, as well as with half $E_{b} / 2$ and third $\mathrm{E}_{\mathrm{b}} / 3$ energy fractions, will slow-down due to collisions with the bulk electrons and ions. The plasma electron density, $n_{e}$, and temperature, $T_{e}$, initially have the greatest impact on beam slowing-down on electrons. The latter is characterised by the slowing-down time, $\tau_{\text {se. }}$. In addition, the local electron temperature, $T_{e}$, will determine where the beam energy is deposited, i.e. thermal ions or electrons, through the critical energy, $E_{c}$. For energies larger than $E_{c}$ the ions will slow down mainly on thermal electrons. As their energy decreases the slowed down beam ions will scatter on thermal ions and further lose energy by collisions with thermal ions and to smaller extent by electron drag. The impurity content and effective charge of the plasma, $Z_{\text {eff, }}$ also affects pitch angle scattering of the 
beam ions. Some of the ions will lose a significant part of their energy so that they eventually thermalize for energies less than few times $T_{i}$. After averaging over some time interval, the fast ion density, $\mathrm{n}_{\mathrm{fi}}$, and normalised fast ion distribution function as a function of fast ion energy and pitch angle, $f_{f i}(E, \theta)$, can be determined. The bulk, i.e. thermal particles, are being heated by energetic beam ions but they are also a subject to both particle and heat transport, neoclassical and turbulent. Fast ions may also be subject to turbulent transport and interactions with various MHD modes. In the end, all the processes described above will contribute to different extent to the quantities which determine $\mathrm{BT}$ and thermal reactivities and reaction rates.

If RF power is applied in addition to NB injection of $D$, the neutron production will be affected in various ways. Indeed, adding RF power to NB fuelled/heated D plasma can have an impact on a number of plasma parameters: for instance ion and electron temperatures, $T_{i}$ and $T_{e}$. In turn, electron temperature changes can affect beam deposition, slowing down and fast ion distributions hence $B T$ reactions, while $T_{i}$ has an indirect (via fast ion slowing down) and direct (via changes in < $\sigma . v\rangle_{\text {Th }}$ and $\langle\sigma . v\rangle_{B T}$ ) impact on thermal and BT rates. RF will also require a minority specie which could affect $D$ bulk ions' density and thus cause fuel dilution. In the investigated (H)D minority scheme, the $\mathrm{H}$ minority will be heated at $\mathrm{n}=1$ fundamental cyclotron frequency, while the bulk $\mathrm{D}$ ions will be heated via collisions with minorities. The fast ion distribution function of $D$ beam ions will be also affected by the RF power directly as fast $D$ beam ions can also absorb energy directly from the $\mathrm{RF}$ wave at $\mathrm{n}=2$ harmonic cyclotron frequency providing wave-particle resonance condition is satisfied. Thus, the $D$ beam ion distribution function can be modified by the RF electric field, which in terms of kinetic description, i.e. solving the Fokker-Plank equation, can be described by means of a quasilinear diffusion coefficient, $D_{Q L}$. For RF wave with frequency near $n=2$ harmonic ion cyclotron $D$ resonance, $\mathrm{D}_{\mathrm{QL}}$ modifies the fast ion distribution function as a high energetic tail is created in the plasma core for energies $E \geq E_{b} \approx 120 \mathrm{keV}$. The plateau region of FI DF, for energies $E \geq E_{b} / 3 \approx 40 \mathrm{keV}$ and $\mathrm{E} \leq \mathrm{E}_{\mathrm{b}} \approx 120 \mathrm{keV}$, is modified as well.

Assuming typical JET conditions, with lon Cyclotron Resonance Heating (ICRH) related parameters $\mathrm{E}$. $/ \mathrm{E}_{+} \approx 2.4, \mathrm{k}_{\perp} \approx 50 \mathrm{~m}^{-1}, \mathrm{f}_{\mathrm{RF}}=42 \mathrm{MHz}$ and magnetic field $\mathrm{B}_{\mathrm{t}}=2.75 \mathrm{~T}$, a resonance with $\omega_{\mathrm{CD}} \approx 2 \pi 21 \mathrm{MHz}$ is produced in the centre of the plasma, giving a maximum of the quasilinear diffusion coefficient, $D_{Q L}$, for $n=2$ harmonic heating of $D$ at about $1 \mathrm{MeV}$, figure $4 \mathrm{a}$ ). It is $80 \%$ lower for NB fast ion full energies, $E_{b} \approx 120 \mathrm{keV}$ and it is zero for thermal particles. This means that the RF electric field will interact with fast ions by pulling a tail in FI DF for energies $E>E_{b}$. In addition to that, as $D_{Q L}$ is finite in the region of beam fast ion energies changes to FI DF plateau will be expected as well. In contrast to this for $\mathrm{H}$ minority, the maximum of the quasilinear diffusion coefficient for $\mathrm{n}=1$ fundamental heating is at about $200 \mathrm{keV}$ and it is slightly lower for thermal $\mathrm{H}$ particles, figure $4 \mathrm{a}$ ). 


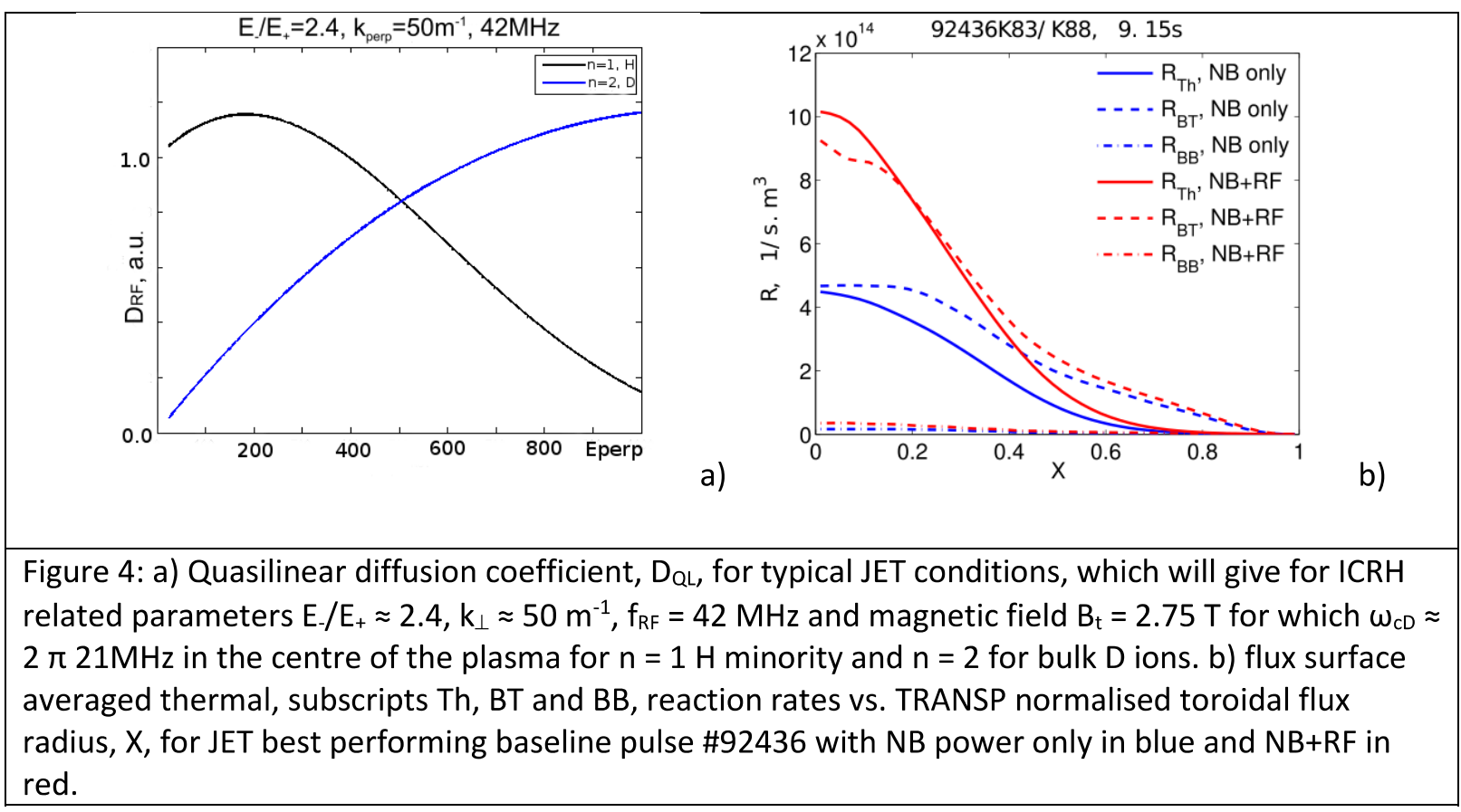

The thermal, BT and BB reaction rates profiles for two cases, with and without RF power in a typical JET pulse calculated by TRANSP [34-37] are shown in figure $4 \mathrm{~b}$ ). In both cases, NB only and NB+RF, thermal and $B T$ rates are peaked in the very core and small for $X>0.4-0.5$. For example, in the case discussed in figure 4 b) BT rates inside $X<0.5$ provide about $67 \%$ of the total BT neutrons, whilst the thermal rates inside this volume are even higher, about $84 \%$ of the total thermal neutrons. BB rates are insignificant, usually two orders of magnitude smaller than BT rates, and therefore they will be ignored in the study. Figure $4 \mathrm{~b}$ ) shows that RF power affects both thermal and BT rates making them larger (red curves) and even more peaked in the centre of the plasma.

In the core the resonant RF wave creates a high energy tail with energies $E>120 k e V$ in the fast ion distribution function and this will affect $\mathrm{BT}$ reactivity $\langle\sigma . \mathrm{V}\rangle_{\mathrm{BT}}$. An example of monoenergetic beam BT reactivity $<\sigma . V\rangle_{\text {BT_MEB }} v$ s. beam energy $E$, fast ion distribution function $\mathrm{F}_{\mathrm{fi}}(E)=\mathrm{n}_{\mathrm{fi}} \mathrm{f}_{\mathrm{fi}}(\mathrm{E})$ representative of a $2 D$ cell in the plasma centre, their product, $<\sigma . v\rangle_{B T}{ }_{-M E B} F_{f i}(E)$, and $B T$ cumulative reaction rate $\mathrm{R}_{B T}(E)$ for NB only (blue lines) and NB+RF (red lines) heating is shown in figure 5. As expected $B T$ reactivity $<\sigma . V\rangle_{\text {BT_MEB }}$ increases with beam energy, however, this increase is relatively small for energies $E>300 \mathrm{keV}$ compared to the range $E=50-300 \mathrm{keV}$, figure $5 \mathrm{a}$ ). The fully evolved fast ion distribution function $\mathrm{F}_{\mathrm{fi}}(\mathrm{E})=\mathrm{n}_{\mathrm{fi}} \mathrm{f}_{\mathrm{fi}}(\mathrm{E})$ provided by TRANSP for time interval larger than a few slowing down times, and the Maxwellian distribution function, $F_{T h}$, in figure 5 b) both calculated at the same point, show the energy at which the bulk provides more particles than NB to interact with RF wave. Clearly for $T_{i}$ in the range 6-8.4 keV and for fast ion energies $E>50 \mathrm{keV}$ there are more $D$ beam ions to interact with RF wave than D ions from the bulk Maxwellian distribution, figure $5 \mathrm{~b}$ ). This might seem to contradict to the widely accepted approximation that RF absorption by given particles depends on particle pressure, $\mathrm{n} \mathrm{T}$. However, this relies on the assumption that the distribution function is Maxwellian, which is not valid for fast ion distribution function studied here. High energetic tail associated with $R F$ is clearly present for energies greater than $E>E_{b} \approx 120 \mathrm{keV}$, red line in figure $5 \mathrm{~b}$ ). The origin of this tail is clearly due to fast $D$ beam ions. One should also note that RF power modifies the plateau of $F_{f i}(E)$, with energies in the range $E>E_{b} / 3 \approx 40 k e V$ to $E<E_{b} \approx 120 k e V$ and these changes are 
essential in BT reaction rate enhancement as it can be seen from $\langle\sigma . v\rangle_{\text {BT_MEB }} F_{f i}$ versus $E$ graph in figures $5 \mathrm{c}$ ) and d). The $B T$ reaction rate $R_{B T}(E)$ in figure $5 d$ ) is presented as a function of $E$, where $E$ is the upper limit of the cumulative integral, i.e. $R_{B T}(E)=n_{D} n_{f i}\langle\sigma . v\rangle_{B T}(E)=$ $n_{D} \int_{0}^{E}\langle\sigma . v\rangle_{B T_{-} M E B} F_{f i}\left(E^{\prime}\right) d E^{\prime}$. Thereafter, we will refer to $\mathrm{R}_{\mathrm{BT}}(\mathrm{E})$ as $\mathrm{BT}$ cumulative reaction rate to distinguish from the conventional definition of $B T$ reaction rate $R_{B T}=R_{B T}(E \rightarrow \infty)$. Fast ions with energies up to about $120 \mathrm{keV}$ are the main contributor in $\mathrm{R}_{\mathrm{B}}$ : in NB only case (blue lines in figures 5 c) and d)) $98 \%$ of neutrons originate from $B T$ reactions with fast ions with energies $E<E_{b} \approx 120 \mathrm{keV}$, while in the NB+RF example (red lines in figures $5 \mathrm{c}$ ) and d)) approximately $2 / 3$ of $\mathrm{R}_{\mathrm{BT}}$ is due to fast ions with $\mathrm{E}<\mathrm{E}_{\mathrm{b}} \approx 120 \mathrm{keV}$. In the latter case the FI DF tail contributes $1 / 3$ to the $\mathrm{BT}$ rates.

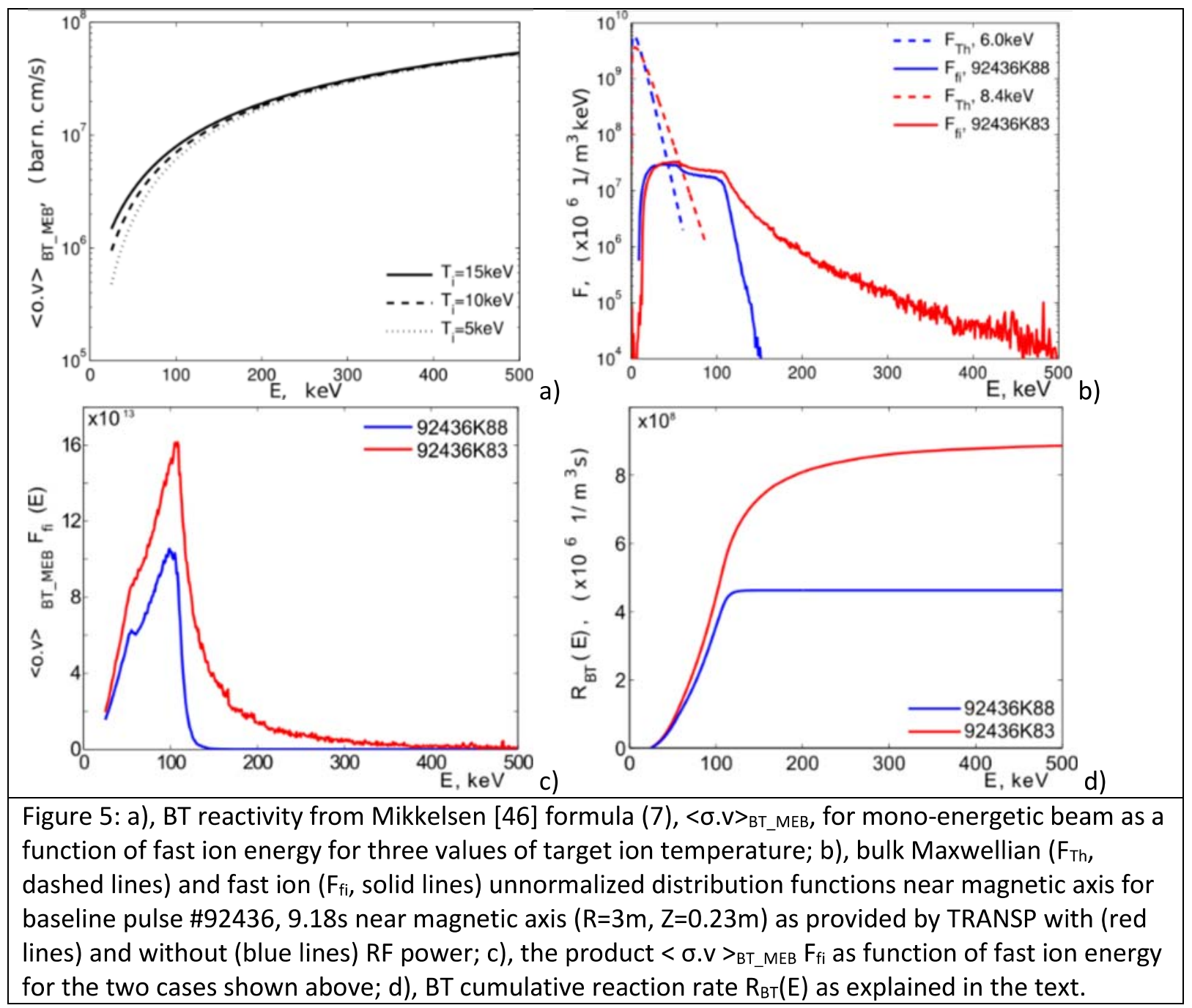

Figure $5 \mathrm{~b}$ ) shows that in the plasma core the RF power will clearly impact on FI DF tail and plateau. Which of these will be affected more depends on the plasma and RF parameters and RF heating scenario. Detailed assessment requires self-consistent modelling including NB and RF heating and particle and heat transport. Modification to FI DF plateau and tail will have a different impact on BT and thermal neutrons and hence on the fusion performance.

For comparison, the impact of the electron and ion temperatures, $T_{e}$ and $T_{i}$, in the NB only case in figure 5 has been studied. Reduction of any of $\mathrm{T}_{e}$ or $\mathrm{T}_{i}$ by about $30 \%$ from the reference will result in 
reaction rate drop of $15 \%$. Decrease in $\mathrm{T}_{\mathrm{e}}$ for instance will affect the plateau region and to a smaller extent the tail of FI DF in the plasma core. The plateau in FI DF will go up with $T_{e}$, which will lead to an increase in BT neutron rates. Lower $T_{i}$ is expected to impact on the FI DF plateau and tail hence BT rates in addition to the thermal rates. The changes in the FI DF tail by varying $T_{e}$ and $T_{i}$ are always negligible in comparison with RF generated tail in FI DF, red line in figure 5 b). This way, by using the product $\langle\sigma . v\rangle_{\text {BT_MEB }} F_{f i}$ as a function of fast ion energy, $E$, and the cumulative reaction rates as the ones shown in figure $5 \mathrm{c}$ ) and d) one can easily assess the contribution by the RF generated tail in $\mathrm{FI}$ DF from supplementary effects of $T_{e}$ and $T_{i}$ on FI DF. For this purpose, graphs as in figure $5 c$ ) and d) will be extensively used for the analysis presented in the next sections.

\section{Results}

Results of the predictive modelling are discussed first in detail in the next section. They provide a basis for power scan studies presented subsequently. Fully predictive modelling of the reference base line and hybrid pulses was performed initially to validate the transport model used here versus the available experimental data. This model is then used in the power scans studies, which provide the fast ion distribution function evolution with NB and RF heating power.

\subsection{Predictive modelling of stationary phases}

In JETTO, electron density, $\mathrm{n}_{e}$, electron and ion temperatures, $\mathrm{T}_{e}$ and $\mathrm{T}_{\mathrm{i}}$, were modelled predictively by means of the Bohm-gyroBohm model [29]. Although the progress in modelling with first principle models is significant [25], [52] the preferred model in this study is the Bohm-gyroBohm model because it provides simplicity and in addition it does not require adjustment of various parameters and settings as many of the first principle models do. In power scans studies, as the ones reported here, keeping transport model settings fixed is essential and it was regarded that the BohmgyroBohm model is best suited for this purpose. H-mode physics is treated with JETTO's own edge barrier transport model, while ELMs were emulated with ELM criteria model. The FRANTIC code was used to treat neutrals. Effective $Z_{\text {eff }}$ is assumed radially constant with time variations as provided by the available spectroscopic diagnostics whereas the main impurity specie is assumed to be $\mathrm{Ni}(\mathrm{Z}=28$, $A=59)$. The radiated power is taken from the available bolometric measurements. In this way, electron and ion sources were calculated self-consistently with the NB and RF heating and equilibrium kinetic plasma profiles.

Fast ion distribution functions and reaction rates are further extracted from a supplementary TRANSP interpretive run. For this purpose, TRANSP was run interpretively with kinetic profiles as used in the corresponding JETTO simulation. The local fast ion distribution functions and BT reactivities are thus available from TRANSP/NUBEAM for analysis and calculation of cumulative reaction rates.

Time traces of calculated and measured total neutrons stored plasma energy, $\mathrm{W}_{\mathrm{p}}$, and total neutron rate, $\mathrm{N}_{\text {TOT, }}$, are provided in figure 6 . Both these quantities are extremely sensitive to $T_{i}$ and fast ion density and energies. For that reason, achieving a good agreement between modelled and experimental data as shown in figure 6 provides important validation of the transport models. 


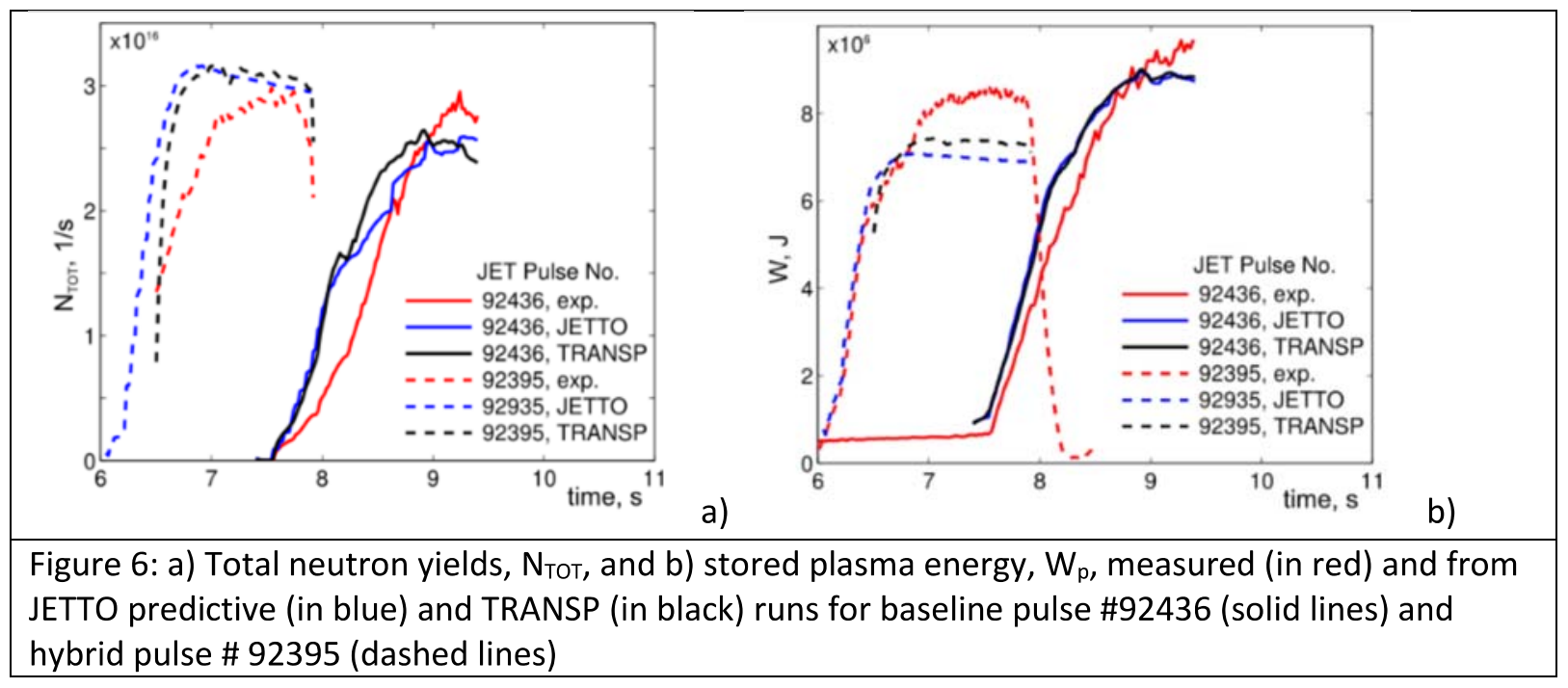

The two codes, JETTO and TRANSP, agree reasonably well between them in calculated total neutrons, $\mathrm{N}_{\text {TOT }}$; less than $1 \%$ for \#92436 (averaged in 8.5-9.4s) and \#92395 (averaged in 7-7.5s). As for the plasma energy, $W_{p}$, we have a discrepancy of about $1 \%$ between the two codes for \#92436 and about $5 \%$ for \#92395, figure $6 \mathrm{~b}$ ). The consistency between JETTO and TRANSP results should come as no surprise as in both cases identical kinetic profiles were used.

The agreement between the simulated (JETTO and TRANSP) and the experimental data for the neutron yield is $8 \%$ for the baseline pulse \#92436. Measured neutrons, $\mathrm{N}_{\text {TOT, }}$, are $10 \%$ lower compared to TRANSP result for \#92395 and 8\% lower than the JETTO prediction, figure 6 a). Agreement with $W_{p}$ data is reasonable, about $5 \%$ between measured and calculated for $\# 92436$. For the hybrid pulse $\# 92395$ the measured $W_{p}$ is $11 \%$ higher compared to TRANSP result and $15 \%$ larger compared to for JETTO, figure $6 \mathrm{~b}$ ).

The simulation results for electron density and temperature and ion temperature profiles of baseline pulse \#92436 are shown in figures 7 a)-c) respectively. Experimental profiles are provided for comparison as well.

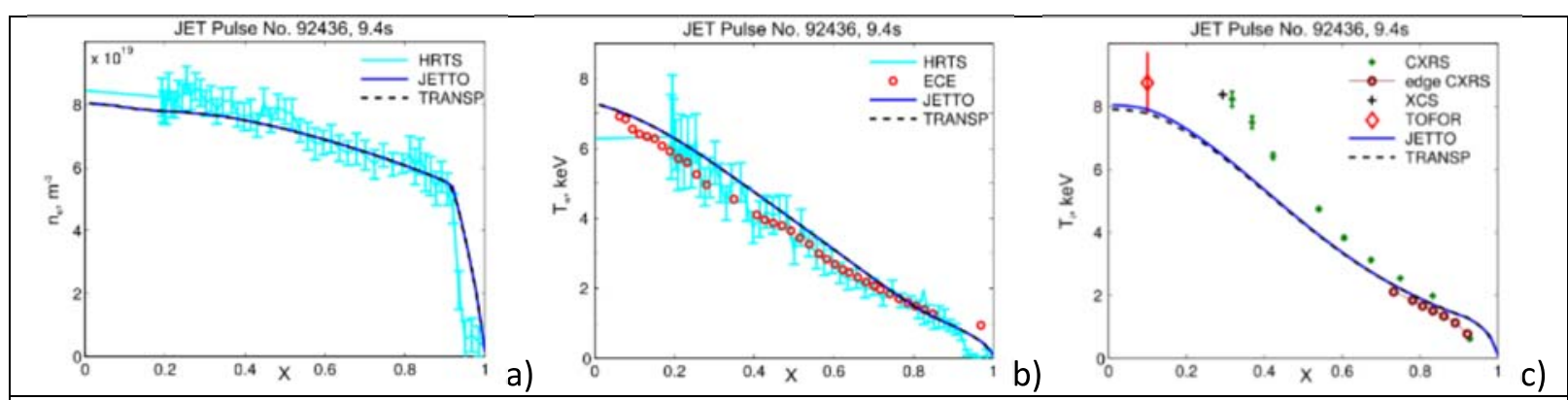

Figure 7: Baseline pulse \#92436, $n_{e}, T_{e}, T_{i}$ profiles at $9.4 s$ from JETTO predictive modelling with BgB model compared to the experimental profiles from the available diagnostics. TRANSP was run with profiles provided by JETTO.

Electron temperature, $T_{e}$, was overestimated at the mid-radius in the simulations, while $T_{i}$ was in good agreement with pedestal $C X$ data for $X>0.7$. Various additional $T_{i}$ measurements were used to constrain the analysis in the plasma core. Standard core CXRS measurements on JET were not of 
satisfactory quality, so data from the NeX CXRS line (shown in figure $7 \mathrm{c}$ )) were used instead. Additional estimates of the central $T_{i}$ was available through crystal spectrometer (XCS) and TOFOR diagnostics, figure $7 \mathrm{c}$ ), and the simulations are in reasonable agreement with these estimates. It is worth noting that the core $\mathrm{T}_{\mathrm{i}}$ is strongly coupled to the edge transport barrier parameters. Higher core $T_{i}$ as suggested by the measurements, however, leads to a poor agreement with neutron rates and plasma energy. In general, achieving perfect match between modelled and all available experimental data, $n_{e}, T_{e}, T_{i}$ profiles and $N_{T O T}$ and $W_{p}$ time traces is practically impossible so results presented in figure 7 are considered a reasonable compromise regarding the available experimental data.

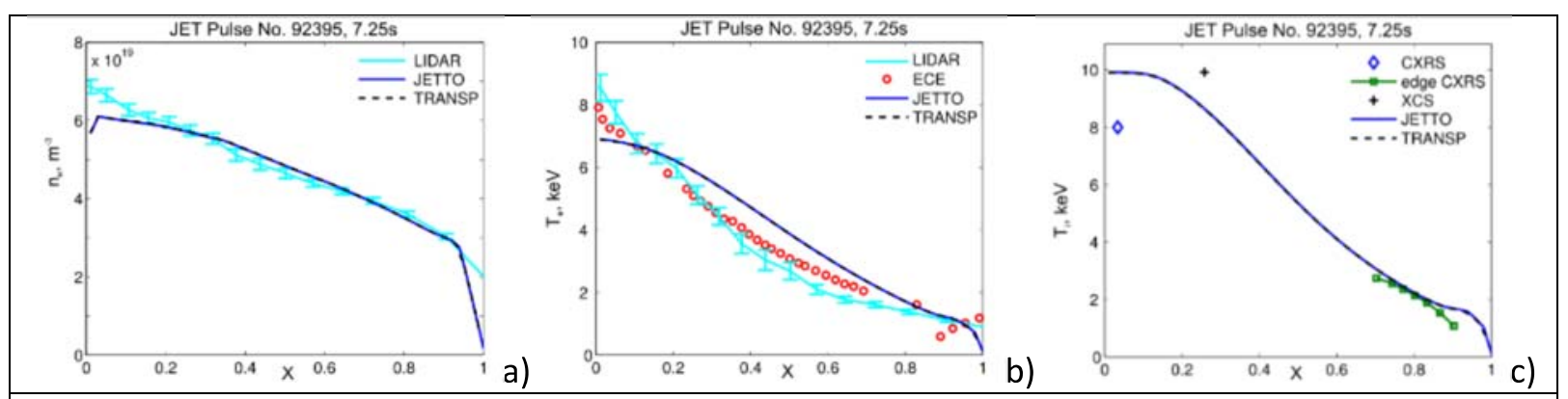

Figure 8: Hybrid pulse \#92395, $n_{e}, T_{e}$ and $T_{i}$ profiles at 7.25s from JETTO and TRANSP interpretive analysis compared to the experimental profiles from the available diagnostics.

Similar results were obtained for the high performance hybrid pulse \#92395, figure 8. Here again, the simulated electron temperature, $T_{e}$, was overestimated at the mid-radius, for $0.2<X<0.7$. The modelled central electron temperature is $13 \%$ lower than the ECE measurements, while for $\mathrm{X}=0.5$ it is about $20 \%$ higher. Changes in Bohm and gyroBohm scaling factors were unable to match better the experimental data. In addition, predictive simulations with a first principles physics model [47] were tried and successfully reproduced $\mathrm{T}_{\mathrm{e}}$ evolution; however, this was achieved at the expense of quality of $n_{e}$ and $T_{i}$ profiles. It was deemed that the small inconsistency in $T_{e}$ by Bohm-gyroBohm model, figure $8 \mathrm{~b}$ ), will only contribute marginally to the neutron rates. Assessment of the impact of $\mathrm{T}_{\mathrm{e}}$ on neutron rates for NB heated plasma is provided at the end of Section 4. It is worth noting that despite the small differences in measured and calculated $T_{e}$ profiles the Bohm-gyroBohm model produces reasonably well the neutron rates in the investigated time interval, figure 6 a). Ion temperature data for the hybrid pulse, \#92395, are shown in figure $8 \mathrm{c}$ ). The standard CXRS diagnostic provided only a single point near the core, $X \approx 0.05$, which is in a reasonable agreement with data from Bell lines (not shown in the graph). Predicted $T_{i}$ by JETTO is higher and in reasonable agreement with crystal spectrometer data (XCS). The agreement with pedestal CX diagnostic is also reasonable.

The profiles from JETTO predictive models were then used in TRANSP simulations to assess fast ion contributions to neutron yields. The combined use of two codes, JETTO and TRANSP, intended to maintain self-consistency of the simulations and provide detailed insight into fast ion physics. JETTO with coupled PION/PENCIL provides self-consistent kinetic profiles and NB and RF power depositions. TRANSP with NUBEAM coupled to TORIC code was run interpretively with fixed profiles as from JETTO. The advantage of this approach is two-fold. First, it allows us to deduce fast ion distribution functions, which in turn were used to analyse reactivity' and reaction rate dependencies 
on fast ion energies. Secondly, the NUBEAM code contains more detail than PENCIL as it treats more accurately the fast ion confinement and orbit effects.

The output of fast ion distribution functions from TRANSP is further validated versus neutron spectra analysis by a synthetic TOFOR diagnostic. The analysis of the DD neutron times-of-flight is discussed below.

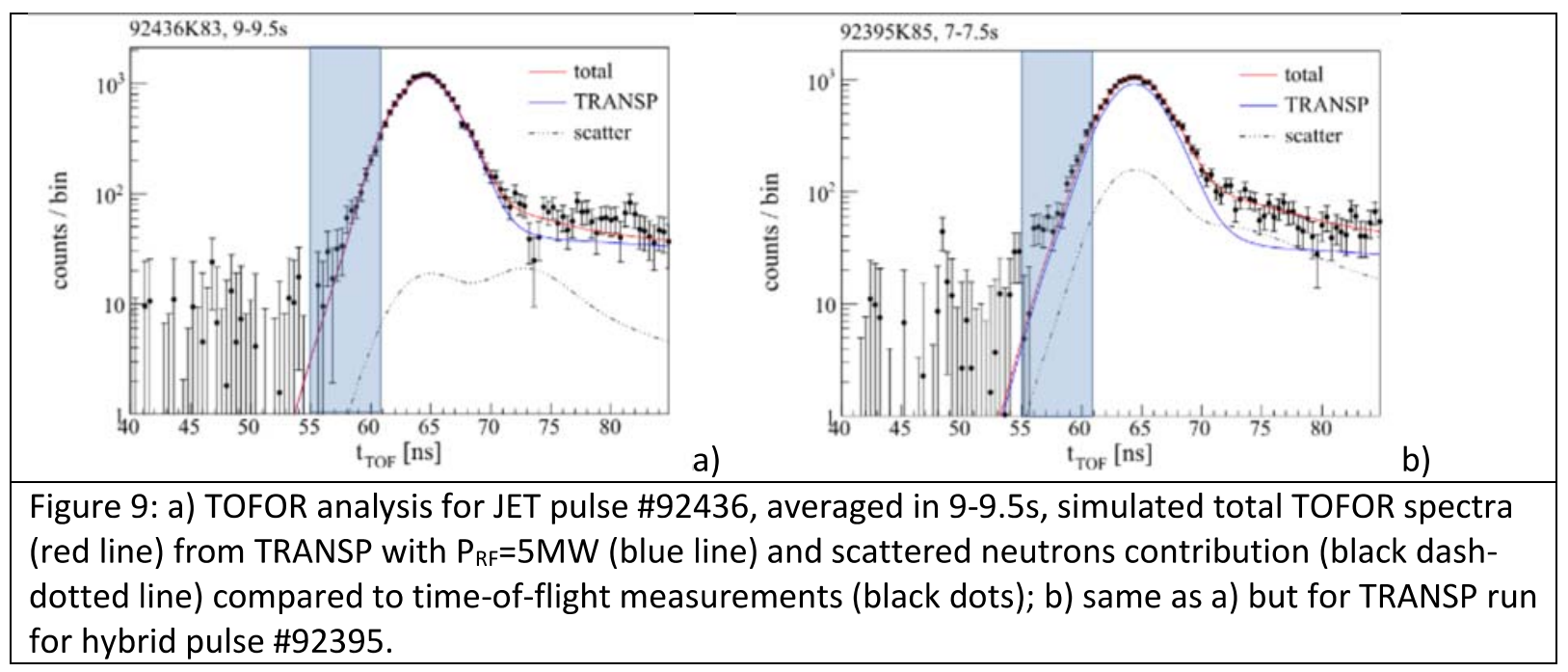

TRANSP data for the fast ion distribution function from pulse \#92436 were used in TOFOR analysis and results of simulated and measured time-of-flight are shown in figure $9 \mathrm{a}$ ), while data from pulse \#92395 are shown in figure $9 \mathrm{~b}$ ). Overall, the agreement is reasonable. The match to the experimental data for baseline \#92436 is good for the whole region of interest with $55 \mathrm{~ns}<\mathrm{t}_{\mathrm{TOF}}<61 \mathrm{~ns}$, figure $9 \mathrm{a}$ ), validating the fast ion distribution function produced by TRANSP. For the hybrid pulse, \#92395, again good agreement was observed for time-of-flight between $57 \mathrm{~ns}$ and $61 \mathrm{~ns}$. The region with flight times smaller than 57ns, however, is systematically underestimated by TRANSP, indicating that TOFOR detects more RF accelerated deuterons than TRANSP predicts.

\subsection{Power scans}

Two types of power scans are discussed here. First one is by varying the input NB and RF power by a small amount ( $4 \mathrm{MW}$ ) compared to the total input power (33MW) and analysing the response of BT and thermal rates and the fast ion distribution function. The second scan is performed by switching off the RF power in cases with and without changes to the kinetic plasma profiles. The aim of the latter is to distinguish contributions from the synergistic effects from the supplementary effects accompanying the application of RF power.

Changes to the fast ion distribution functions and resulting cumulative neutron rates by varying the $\mathrm{NB}$ and RF power are accounted for by modifying the auxiliary power by about $4 \mathrm{MW}$, which for the reference cases of JET pulses \#92436 and \#92395 means 15\% for NB and 70-80\% for the RF power. The latter implies RF power of about 9-9.5 MW which is somewhat above the present capabilities of JET ICRH plant. The focus of the analysis is, however, not to extrapolate fusion performance with auxiliary power rather than to study the impact of the NB and RF power on the fast ion distribution function and this is only possible if the NB and RF power are varied in equal amounts. The applied NB power was varied between $23 \mathrm{MW}$ and $32 \mathrm{MW}$ by changing the neutral beam injection energy while 
keeping all the available beam lines. The advantage of this approach is that the geometrical parameters of the beam lines are kept unchanged during simulations. NB and RF powers have different impacts on the fast ion distribution function, figure 5 , as the former is expected to impact mainly in the plateau region 40-120keV, while RF power is expected to pull an energetic tail for $\mathrm{E}>120 \mathrm{keV}$. Performing the power scan by varying $\mathrm{P}_{\mathrm{NB}}$ and $\mathrm{P}_{\mathrm{RF}}$ will also show in what energy range changes to the fast ion distribution function are more beneficial regarding fusion performance.

Two additional cases without RF power are considered as well; one with plasma parameters corresponding to $\mathrm{P}_{\mathrm{RF}}=0$ predictive run, the other without $\mathrm{RF}$ heating but with kinetic profiles as from the reference case for which $\mathrm{P}_{\mathrm{RF}} \neq 0$. The aim of this comparison is to separate impact of the sheer synergistic effects from supplementary effects resulting from the application of RF power as for instance the impact of $T_{e}$ and $T_{i}$ changes with $P_{R F}$.

Reasonably good modelling data matching well the experimental measurements and kinetic profiles as seen in figures 6, 7 and 8 confirm the utilisation of Bohm-gyroBohm model in the predictive studies. Changing the heating power however may have indirect impact on several parameters which could affect the particle and heat transport. For instance, it is known [48] that large $P_{\mathrm{RF}}$ can mitigate the impurity accumulation and hence the radiated power. On the other hand, too small RF power could also produce more heavy impurities due to sheath effects. This contribution was not accounted for in the power scan reported here. Another example is $\mathrm{H}$-mode pedestal pressure scaling with input power. In our study we use an empirical expression based on a global confinement and pedestal database [49]. Recent studies [50] of JET ILW database has found that the scaling of the pedestal stored energy with heating power is only slightly lower than that used in [49]. Providing a small change in the heating power is used, about $12 \%$, and due to the fact that power dependence is weak the two studies [49], [50] agree reasonably well, within about $1 \%$, on the predicted pedestal energy. Therefore, using formula (2) from Cordey et al [49] and assuming unchanged Top Of the Barrier (TOB) density provides a suitable scaling expression for the electron and ion temperature at TOB. The latter is calculated to be of the order of $0.9-1 \mathrm{keV}$ for the refence cases, while varying NB or $\mathrm{RF}$ power by $4 \mathrm{MW}$ results in pedestal pressure changes by about $10-15 \%$.

\subsubsection{Neutron yield changes with NB and RF power}

Modifications in parameters related to DD fusion performance with auxiliary heating power for \#92436 are shown in table 1. Table 2 shows the result of power scan for \#92395. In these power scans it was assumed that RF parameters and coupling are unchanged for RF power scans while NB power changes via change in injected beam energy as for the reference cases with real power of about $27 \mathrm{MW}$ neutral beam injection voltage was between $98 \mathrm{kV}$ and $111 \mathrm{keV}$ with average value of $106 \mathrm{kV}$. For $\mathrm{P}_{\mathrm{NB}}=23 \mathrm{MW}$ cases the injected beams were assumed to be at $95 \mathrm{kV}$, while for the high power cases of $31 \mathrm{MW}$ beams voltage of $125 \mathrm{kV}$ was used.

Table 1: Results of power scan simulations of JET baseline pulse \#92436 at 9.15s. NB and RF power are provided in columns 2 and 3 followed by $\mathrm{Fl}$, main $\mathrm{D}$ ion and electron densities and temperatures, BT and thermal averaged reactivities and reaction rates. All these quantities are volume averaged, noted by $\langle>$, inside plasma volume $0<X<0.4$. The last three columns provide the time averaged, 8.9-9.4s, BT, thermal and total number of reactions. The first row in the table gives the absolute values of these quantities for the reference case with actual NB and RF power. Rows \#1 to \#6 show the relative changes of the quantities with respect to the reference case. Bottom two rows, $\# 5$ and \#6, are both for $P_{R F}=0$ but row \#5 is with plasma parameters corresponding to $\mathrm{P}_{\mathrm{RF}}=0$ while \#6 is with plasma parameters as in the reference case. Changes larger than $7 \%$ are in bold. 


\begin{tabular}{|c|c|c|c|c|c|c|c|c|c|c|c|c|c|c|}
\hline \#92436 & $\begin{array}{l}\mathrm{P}_{\mathrm{NB}}, \\
\mathrm{MW}\end{array}$ & $\begin{array}{l}\mathrm{P}_{\mathrm{RF}} \\
\mathrm{MW}\end{array}$ & $\begin{array}{l}\left\langle\mathrm{n}_{\mathrm{fi}}\right\rangle, \\
\mathrm{m}^{-3} \\
\end{array}$ & $\begin{array}{l}\left\langle\mathrm{n}_{\mathrm{D}}\right\rangle \\
\mathrm{m}^{-3} \\
\end{array}$ & \begin{tabular}{|l}
$\left.<n_{e}\right\rangle$ \\
$m^{-3}$ \\
\end{tabular} & $\begin{array}{l}\left\langle\mathrm{T}_{\mathrm{e}}>\right. \\
\mathrm{keV}\end{array}$ & $\begin{array}{l}\left\langle<\mathrm{T}_{\mathrm{i}}\right\rangle \\
\mathrm{keV}\end{array}$ & $\mid \begin{array}{l}\left.\langle<\sigma \mathrm{V}\rangle_{\mathrm{BT}}\right\rangle, \\
\mathrm{m}^{3} / \mathrm{s}\end{array}$ & $\mid \begin{array}{l}\left.\langle\sigma \sigma \mathrm{V}\rangle_{\mathrm{Th}}\right\rangle, \\
\mathrm{m}^{3} / \mathrm{s}\end{array}$ & $\begin{array}{l}\left\langle R_{\mathrm{BT}}\right\rangle \\
1 / \mathrm{m}^{3} \mathrm{~s}\end{array}$ & $\begin{array}{l}\left\langle R_{T h}\right\rangle \\
1 / \mathrm{m}^{3} \mathrm{~s}\end{array}$ & $\begin{array}{l}N_{B T}, \\
1 / \mathrm{s}\end{array}$ & $\begin{array}{l}N_{\text {Th }} \\
1 / \mathrm{s}\end{array}$ & $\begin{array}{l}N_{\text {TOT }} \\
1 / \mathrm{s}\end{array}$ \\
\hline reference & 27.5 & 5.7 & $2.53 \mathrm{E}+18$ & $7.05 \mathrm{E}+19$ & $7.51 \mathrm{E}+19$ & 5.799 & 6.647 & $3.26 \mathrm{E}-24$ & $2.13 \mathrm{E}-25$ & $5.70 \mathrm{E}+14$ & $4.92 \mathrm{E}+14$ & $1.48 \mathrm{E}+16$ & $9.77 \mathrm{E}+15$ & $2.52 \mathrm{E}+16$ \\
\hline$\# 1$ & 23.4 & 5.7 & 0.75 & 1.00 & 0.99 & 0.90 & 0.86 & 0.81 & 0.67 & 0.61 & 0.67 & 0.64 & 0.66 & 0.64 \\
\hline$\# 2$ & 31.6 & 5.7 & 1.37 & 1.02 & 1.02 & 1.07 & 1.11 & 1.25 & 1.31 & 1.74 & 1.34 & 1.70 & 1.35 & 1.57 \\
\hline$\# 3$ & 27.5 & 1.7 & 0.89 & 1.02 & 1.02 & 0.83 & 0.84 & 0.80 & 0.62 & 0.72 & 0.64 & 0.79 & 0.64 & 0.73 \\
\hline$\# 4$ & 27.5 & 9.7 & 1.13 & 0.98 & 0.98 & 1.15 & 1.09 & 1.25 & 1.24 & 1.39 & 1.18 & 1.26 & 1.20 & 1.24 \\
\hline$\# 5$ & 27.5 & 0 & 0.84 & 1.03 & 1.03 & 0.75 & 0.73 & 0.73 & 0.39 & 0.62 & 0.42 & 0.69 & 0.43 & 0.59 \\
\hline$\# 6$ & 27.5 & 0 & 0.96 & 1 & 1 & 1 & 1 & 0.89 & 1 & 0.85 & 1 & 0.91 & 1 & 0.95 \\
\hline
\end{tabular}

Reference cases correspond to the actual plasma pulses, \#92436 and \#92395, as modelled predictively by JETTO and interpretatively by TRANSP with results shown in figures 6,7 and 8 . NB and RF power variations in cases \#1 to \#6 are shown in columns 2 and 3 followed by fast ions, main ions and electron densities and temperatures, BT and thermal averaged reactivities and reaction rates. They are all volume averaged inside plasma volume $0<X<0.4$, where fusion reactions predominantly happen, see figure $4 \mathrm{~b}$ ). Changes in $\mathrm{BT}$ and thermal reactivities and rates are taken from TRANSP at given time slice. TRANSP does not provide thermal reactivity, $\langle\sigma . v\rangle_{\text {Th }}$, so an assessment of it is derived by dividing the reaction rate $\left\langle\mathrm{R}_{\mathrm{Th}}>\text { to } 2^{*}<\mathrm{n}_{D}\right\rangle^{2}$. BT, thermal and total number of neutron are time averaged in the time interval indicated in the table. The top four rows of the tables give the results for NB and RF power variation by $4 \mathrm{MW}$, while bottom two rows provide results from the $\mathrm{P}_{\mathrm{RF}}=0$ cases discussed above.

Table 2: Same as table 1 but for power scan simulations of JET hybrid pulse \#92395 for time slice at 7.25s. The last three columns provide the time averaged, 7.0-7.5s, BT, thermal and total reactions. Bottom two rows, \#5 and \#6, are both for $P_{R F}=0$ but row \#5 is with plasma parameters corresponding to $P_{R F}=0$ while \#6 is with plasma parameters as in the reference case. Changes larger than $7 \%$ are in bold.

\begin{tabular}{|c|c|c|c|c|c|c|c|c|c|c|c|c|c|c|}
\hline$\# 92395$ & $\begin{array}{l}\mathrm{P}_{\mathrm{NB}}, \\
\mathrm{MW}\end{array}$ & $\begin{array}{l}P_{R F}, \\
M W\end{array}$ & $\begin{array}{l}\left\langle n_{f i}\right\rangle, \\
m^{-3}\end{array}$ & $\begin{array}{l}\left\langle n_{D}\right\rangle \\
m^{-3}\end{array}$ & \begin{tabular}{|l}
$\left\langle\mathrm{n}_{\mathrm{e}}\right\rangle$ \\
$\mathrm{m}^{-3}$
\end{tabular} & $\begin{array}{l}\left\langle\mathrm{T}_{\mathrm{e}}\right\rangle \\
\mathrm{keV}\end{array}$ & $\begin{array}{l}\left\langle\mathrm{T}_{\mathrm{i}}\right\rangle \\
\mathrm{keV}\end{array}$ & $\begin{array}{l}\left.\langle<\sigma \mathrm{VV}\rangle_{\mathrm{BT}}\right\rangle, \\
\mathrm{m}^{3} / \mathrm{s}\end{array}$ & $\begin{array}{l}\left.<<\sigma v\rangle_{T h}\right\rangle, \\
\mathrm{m}^{3} / \mathrm{s}\end{array}$ & $\begin{array}{l}\left\langle R_{B T}\right\rangle \\
1 / \mathrm{m}^{3} \mathrm{~s}\end{array}$ & $\begin{array}{l}\left\langle R_{T h}\right\rangle, \\
1 / \mathrm{m}^{3} \mathrm{~s}\end{array}$ & $\begin{array}{l}N_{B T}, \\
1 / s\end{array}$ & $\begin{array}{l}N_{\text {Th, }} \\
1 / \mathrm{s}\end{array}$ & $\begin{array}{l}N_{\text {TOT, }} \\
1 / \mathrm{s}\end{array}$ \\
\hline reference & 26.8 & 5.2 & $5.24 \mathrm{E}+18$ & $5.07 \mathrm{E}+19$ & $5.64 \mathrm{E}+19$ & 5.691 & 8.343 & $3.63 \mathrm{E}-24$ & 4.07E-25 & $9.73 \mathrm{E}+14$ & $4.88 \mathrm{E}+14$ & $1.98 \mathrm{E}+16$ & $9.34 \mathrm{E}+15$ & $3.10 \mathrm{E}+16$ \\
\hline$\# 1$ & 22.8 & 5.2 & 0.80 & 1.00 & 0.98 & 0.93 & 0.89 & 0.83 & 0.75 & 0.66 & 0.76 & 0.67 & 0.75 & 0.69 \\
\hline$\# 2$ & 30.8 & 5.2 & 1.35 & 0.95 & 0.99 & 1.10 & 1.14 & 1.26 & 1.38 & 1.60 & 1.24 & 1.60 & 1.25 & 1.53 \\
\hline$\# 3$ & 26.8 & 1.1 & 0.87 & 1.06 & 1.05 & 0.84 & 0.84 & 0.77 & 0.63 & 0.71 & 0.72 & 0.75 & 0.72 & 0.73 \\
\hline$\# 4$ & 26.8 & 9.3 & 1.12 & 0.95 & 0.96 & 1.14 & 1.09 & 1.27 & 1.25 & 1.36 & 1.12 & 1.30 & 1.14 & 1.27 \\
\hline$\# 5$ & 26.8 & 0 & 0.84 & 1.07 & 1.06 & 0.80 & 0.77 & 0.72 & 0.50 & 0.64 & 0.59 & 0.68 & 0.59 & 0.65 \\
\hline$\# 6$ & 26.8 & 0 & 0.94 & 1 & 1 & 1 & 1 & 0.87 & 1 & 0.81 & 1 & 0.86 & 1 & 0.90 \\
\hline
\end{tabular}

Both NB and RF powers affect insignificantly, less than $6 \%, \mathrm{n}_{\mathrm{D}}$ and $\mathrm{n}_{\mathrm{e}}$ in all simulations presented in tables 1 and 2. The only exception is the case with $P_{R F}=0$ (case \#5) for the hybrid pulse \#92395 in table 2 , where $n_{D}$ increases by about $7 \%$ if RF power is turned off. Having relatively small variations of $n_{D}$ and $n_{e}$ with power is a consequence of setting $n_{e}$ at the TOB unchanged and only scaling $T_{e}$ and $T_{i}$ when varying pedestal pressure with input power. Normally, the plasma density is feedback controlled in experiments, so this assumption is meant to replicate the way in which an experiment will be conducted. Avoiding large variations of $n_{D}$ has another advantage in our analysis as it eliminates the well-known dependences of the reaction rates on this parameter, i.e. linear for BT and square for thermal rates, thus focussing on dependencies related to $T_{e}, T_{i}, n_{f i}$ and fast ion distribution function.

Considering the impact of NB power on fusion performance for both scenarios, rows \#1 and \#2 in tables 1 and 2, one concludes that 4MW of NB power will cause large changes in $\left\langle n_{\mathrm{fi}}\right\rangle, 20-37 \%$, and 
averaged $B$ T reactivity $\langle<\sigma . v\rangle_{B T}>$ changes by $17-26 \%$. Consequently, $B T$ reaction rates $\left\langle R_{B T}>\right.$ are significantly affected by NB power, $34-74 \%$. In addition, the thermal rate $<R_{T h}>$ also changes by about $24-34 \%$ due to the impact of NB on $\mathrm{T}_{\mathrm{i}}$.

RF power impact is shown in rows \#3, \#4 in tables 1 and 2 , and it affects mainly $T_{e}, T_{i}$ and averaged BT reactivity $<<\sigma . v\rangle_{B T}>$ by $20-27 \%$ and reaction rates $<R_{B T}>$ by $29-39 \%$. The latter is accompanied by changes in thermal reactivity $<<\sigma . v\rangle_{\text {Th }}>$ by $24-38 \%$ and rates $\left\langle R_{T h}>\right.$ by $12-36 \%$. RF power will change $\mathrm{n}_{\mathrm{fi}}$ considerably but to a lower extent compared to NB power. The case with zero RF power, case \#5, indicates the total effect of RF power. It shows that increase in neutrons with switching-on the RF power is due to combination of factors: $n_{\mathrm{fi}},\langle\sigma . \mathrm{V}\rangle_{\mathrm{BT}}, \mathrm{T}_{\mathrm{e}}$ and $\mathrm{T}_{\mathrm{i}}$, the latter also directly affecting $\langle\sigma . v\rangle_{\mathrm{Th}}$. All these contributions have comparable influences on neutron rate.

From tables 1 and 2 rows \#1 to \#4 one concludes that 4MW of extra NB power will produce an additional $50 \%$ neutrons. Increasing the RF power by the same amount will only deliver about $25 \%$ more neutrons. The main contributor to this enhancement is the BT rates which are more sensitive to changes in the NB power. Although BT reactivity increases with fast ion energy, figure 5 a), it seems that in the investigated cases the RF power cannot generate enough fast particles to show a significant enhancement. In the conditions of the experiments shown here NB power changes to the plateau of the fast ion distribution function seem to be more beneficial regarding the neutron rates. Changes in thermal rates reflect the changes in $\mathrm{T}_{i}$ with $\mathrm{P}_{\mathrm{NB}}$ and $\mathrm{P}_{\mathrm{RF}}$.

Comparing baseline to hybrid cases, one can conclude that $\mathrm{n}_{\mathrm{fi}}$ changes with varying the heating power in a similar way for both scenarios. The same conclusion can be drawn for $T_{e}$ and $T_{i}$. Although $\mathrm{T}_{i}$ changes are identical the thermal reaction rate changes are different. For instance, from case \#1 it follows that by dropping the NB power by $4 \mathrm{MW}, \mathrm{T}_{\mathrm{i}}$ will go down by $14 \%$ in baseline (table 1 ) and $11 \%$ (table 2 ) for hybrid pulse; however, $\left.\langle<\sigma . v\rangle_{\text {Th }}\right\rangle$ is reduced by $33 \%$ and $25 \%$ respectively. This could be due a number of factors, i.e. profile effects, different refence $T_{i}$ and possibly stronger than square ( $\sim 2.5)$ dependence of $\left\langle\langle\sigma . v\rangle_{T h}\right\rangle$ on $T_{i}$. Interestingly, BT reactivity $\left\langle\langle\sigma . v\rangle_{B T}\right\rangle$ changes with heating power is similar in baseline scenario as it changes by the same amount independently of whether NB or RF power is changed. In hybrid scenario the RF power changes affect $<<\sigma . V\rangle_{B T}>$ slightly more strongly than corresponding NB power variations.

Row \#6 shows the changes in reactivities and rates if RF power is removed, but $n_{e}, T_{e}$ and $T_{i}$ profiles are kept as in the reference case, while row \#5 accounts for changes in these parameters with RF power. The aim of this comparison is to assess to what extent changes in kinetic profiles affect the fusion performance and to differentiate them from synergistic effects due to changes in fast ion distribution function. From the numbers in rows \#5 and \#6 in tables 1 and 2 it can be concluded that the sheer synergistic effects of RF power on neutron yields are moderate for the investigated pulses. Fast ion density changes by only $5 \%$, if RF power is removed but $n_{e}, T_{e}$ and $T_{i}$ profiles kept unchanged, row \#6, while reactivity goes down by about $11-13 \%$ and reaction rates by $15-19 \%$. Total neutron count drops by $5-10 \%$ due to changes in BT neutrons of 9-14\%. In contrast to this, if changes in plasma parameters due to RF are taken into account, row \#5, there is significant drop in both thermal and BT reactivities and reaction rates as shown in tables 1 and 2 . The total neutron count in this case will drop by about $35-41 \%$. Clearly for the two pulses investigated here, the supplementary effect accompanying application of RF power plays an important role in fusion performance. 


\subsubsection{Fast ion densities and distribution function}

The causes of the observed dependencies are further analysed by examining $\mathrm{n}_{\mathrm{fi}}$, beam depositions and heating profiles and fast ion distribution function in the plasma core.

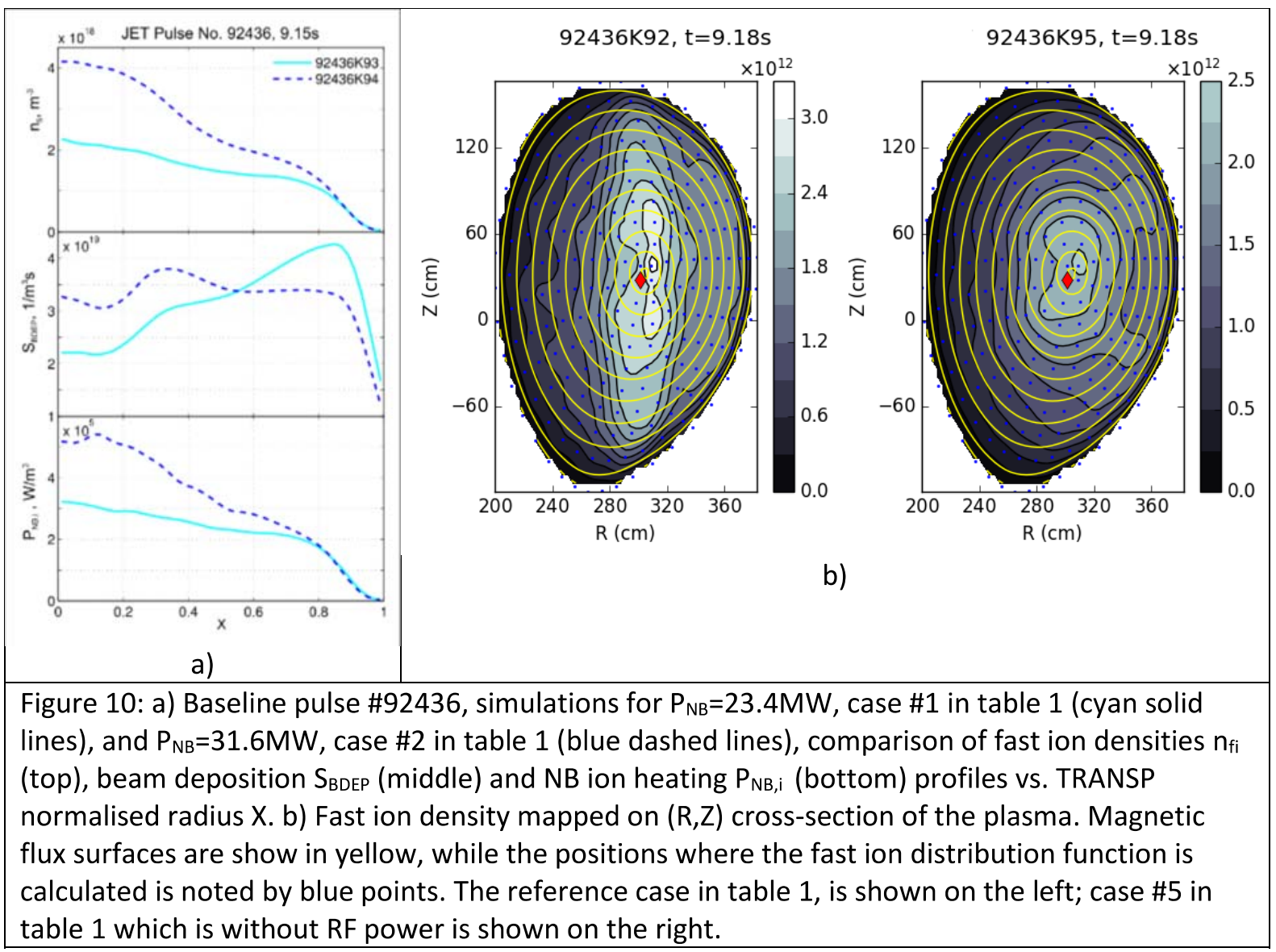

Varying the NB power by $4 \mathrm{MW}$, cases \#1 and \#2 in table 1 , results in significant changes in $\mathrm{n}_{\mathrm{fi}}$, beam deposition and NB ion heating, figure $10 \mathrm{a}$ ). The beam deposition profile changes from off-axis for lower $\mathrm{P}_{\mathrm{NB}}$ and beam energy to a flat profile for $\mathrm{P}_{\mathrm{NB}}=31.6 \mathrm{MW}$.

Comparing the reference case with that without RF power case \#5, one sees similar NB electron and ion heating as well as same beam deposition; however, fast ion density is higher in the core with RF power. The plasma $(\mathrm{R}, \mathrm{Z})$ cross-sections shown in figure $10 \mathrm{~b})$ confirm this conclusion and show distribution of fast ions along the IC resonance when RF power is applied. Poloidal asymmetry of fast ion density is pronounced when RF power is applied. The increase in $\mathrm{n}_{\mathrm{fi}}$ in the core for the reference case compared to zero RF power case is due to RF power pulling high energetic tail and fast particles become less collisional. This conclusion is also confirmed by examining case \#6 in table 1 compared to the reference case. Increase in $\mathrm{n}_{\mathrm{fi}}$ with $\mathrm{RF}$ power in this case is purely due to generating more high energy particles which have larger slowing down time thus increasing $\mathrm{n}_{\mathrm{fi}}$. 


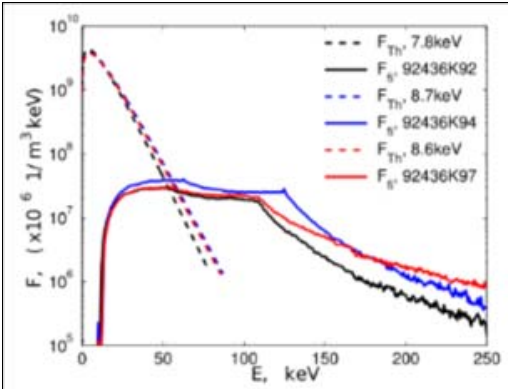

a)

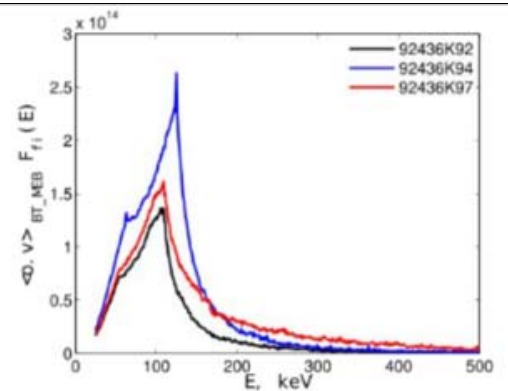

b)

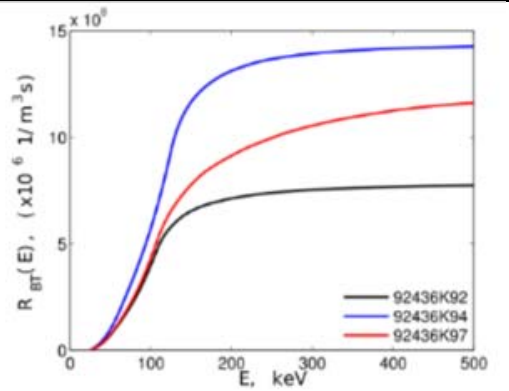

c)

Figure 11: Baseline pulse \#92436, 9.18s, fast ion distribution function, $\mathrm{F}_{\mathrm{fi}}$ (solid lines), and thermal Maxwellian distribution function, $F_{T h}$ (dashed lines ), in a), the product $\langle\sigma . V\rangle_{B T}{ }_{\text {MEB }} F_{f i}(E)$ in $b$ ) and BT cumulative reaction rate $R_{B T}(E)$ in c) near magnetic axis at $R=3 \mathrm{~m}, \mathrm{Z}=0.23 \mathrm{~m}$ (position is also indicated by red dot in figure $10 \mathrm{~b}$ ) for $92436 \mathrm{~K} 92$ case). Reference case is in black, $4 \mathrm{MW}$ higher NB power is in blue and $4 \mathrm{MW}$ higher RF power is shown by red lines.

Changes in fast ion distribution function in the core for baseline scenario \#92436 after increasing or decreasing NB and RF power by $4 \mathrm{MW}$ are shown in figures 11 and 12. Reference case is in black, cases where changes to the NB power is made are in blue, while RF power scans are in red. In addition, the product $\langle\sigma . v\rangle_{\text {BT_MEB }} F_{f i}$ and $B T$ cumulative reaction rate $R_{B T}(E)$ are shown in order to illustrate which part of affected fast ion distribution function impacts most on the reaction rates.

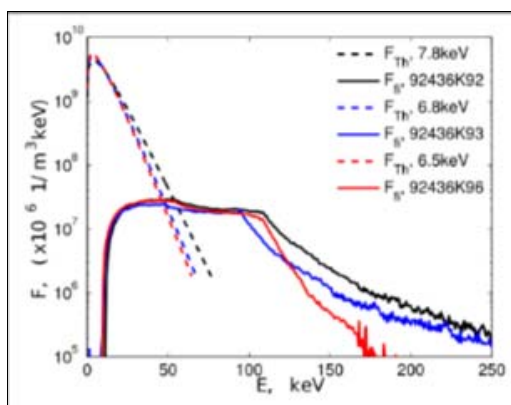

a)

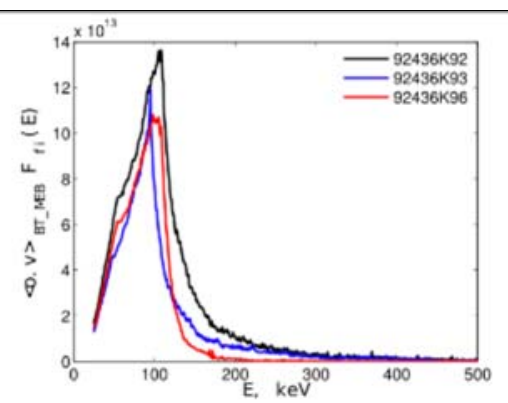

b)

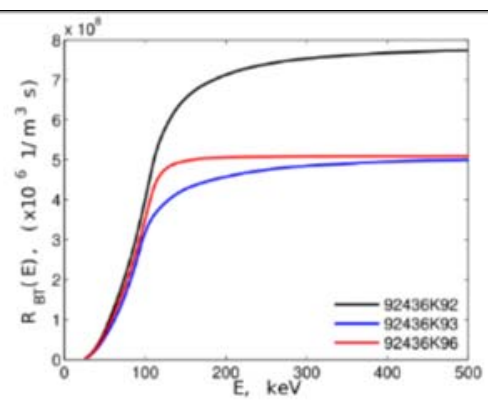

c)

Figure 12: same as the figure 11 but for the case of lowering the NB (blue) and RF (red) power by $4 \mathrm{MW}$.

Following the red lines in figure 12 it is clear that by decreasing the RF power by $4 \mathrm{MW}$ (case \#3 in table 1) fast ion distribution function tail will be largely reduced, red lines in figure $12 \mathrm{a}$ ). This will lead to FI DF tail practically having no contribution to the BT reactivity as seen in figure $12 \mathrm{~b}$ ) with red line nearly zero, while the cumulative integral in figure $12 \mathrm{c}$ ) is constant for energies $E>110 \mathrm{keV}$. It is estimated that about $83 \%$ of the averaged reactivity is provided by fast ions with energies up to $110 \mathrm{keV}$ and only $17 \%$ by fast ions with energies above $110 \mathrm{keV}$. On the other hand, increasing the RF power (case \#4 in table 1) will create a larger tail as seen when comparing the black and red lines in figure 11 a). This will ultimately change $R_{B T}(E)$ for energies above the beam energy, $E_{b}$, as shown in figure $11 \mathrm{~b}$ ). One should note that the latter is now significantly larger and above zero for $E>110 \mathrm{keV}$. It is clear from figure $11 \mathrm{c}$ ) following the red line that contributions from particles with energies in the range $110-500 \mathrm{keV}$ to the BT reactivity is significant, $56 \%$, for RF power increase by $4 \mathrm{MW}$. The total increase of $R_{B T}$ due to RF power in figure $11 \mathrm{c}$ ) is about $48 \%$ which is slightly higher than the flux 
surface averaged value of $39 \%$ in table 1 row \#4. The latter shows that the BT rates enhancement with RF power is localised in the core and to some extent along the IC resonance line, figure $10 \mathrm{~b}$ ).

The impact of the NB power on the fast ion distribution function and BT reaction rate differs qualitatively from the aforementioned case. Comparing the black and the blue lines in the case where NB power was increased by $4 \mathrm{MW}$, figure $11 \mathrm{a}$ ), or reduced by the same amount, figure $12 \mathrm{a}$ ), one sees that the NB power mainly affects the plateau region of the fast ion distribution function, while the slope of the tail is practically unchanged. The latter is determined by the fast ion slowing down, so the FI DF tail slope for $E>E_{b}$ depends on $T_{e}$ and $T_{i}$. The plateau changes in two ways: (i) the high energy end is pushed towards higher energies with beam energy and power and (ii) the plateau is lifted up with NB power. One can conclude in this case that the changes in the BT rate is mainly due to changes in the fast ion distribution function in the beam energy range, up to $95 \mathrm{keV}$ for case $\# 1$ (table 1) with $\mathrm{P}_{\mathrm{NB}}=23.4 \mathrm{MW}, 110 \mathrm{keV}$ for reference case with $\mathrm{P}_{\mathrm{NB}}=27.5 \mathrm{MW}$ and $125 \mathrm{keV}$ for case \#2 with $\mathrm{P}_{\mathrm{NB}}=31.6 \mathrm{MW}$. In all these cases fast ions in the plateau of the distribution function contribute nearly two thirds (67\% for case $\# 2$ to $61 \%$ for case \#1) to the BT reactivity.

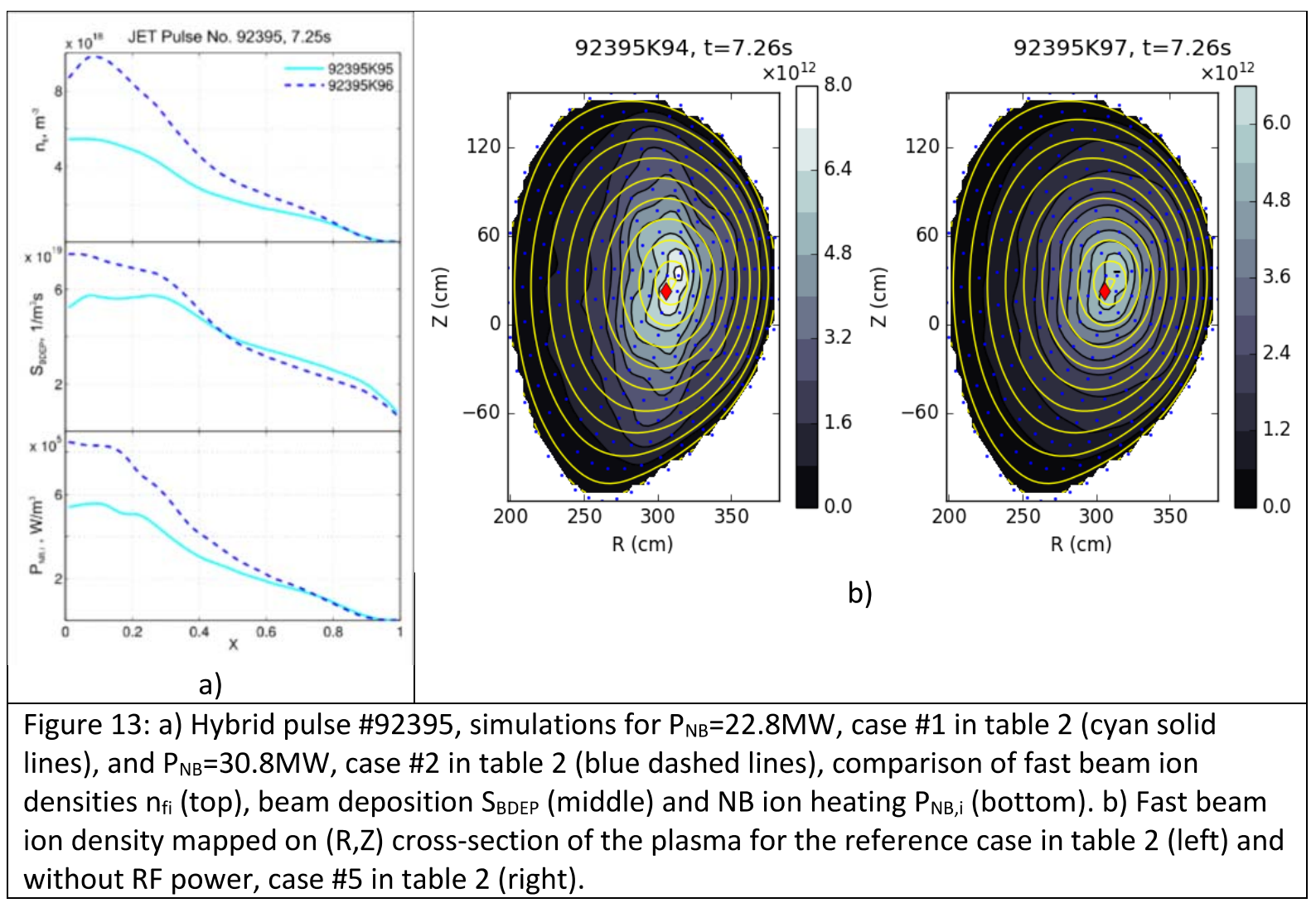

As with baseline, in hybrid pulse \#92935 higher NB power by 4MW, case \#2 in table 2, results in higher electron and ion heating; fast ion density and beam deposition are higher in the core compared to the lower power case with $\mathrm{P}_{\mathrm{NB}}$ reduced by $4 \mathrm{MW}$, figure $13 \mathrm{a}$ ).

Similar NBI electron and ion heating as well as beam deposition were observed when comparing the reference case with no RF power case. Fast ion density is higher in the core with RF power. $(R, Z)$ plasma cross-sections in figure $13 \mathrm{~b}$ ) confirm this conclusion and show enhanced distribution of fast ions along IC resonance when RF power is applied. 
Case \#5 with zero RF power shows that an increase in neutrons commensurate with switching on the RF power is due to a combination of factors: $\mathrm{n}_{\mathrm{fi}},\langle\sigma . v\rangle_{\mathrm{BT}}, \mathrm{T}_{\mathrm{e}}$ and $\mathrm{T}_{\mathrm{i}}$ affecting $\langle\sigma . v\rangle_{\text {Th }}$ all having comparable contributions.

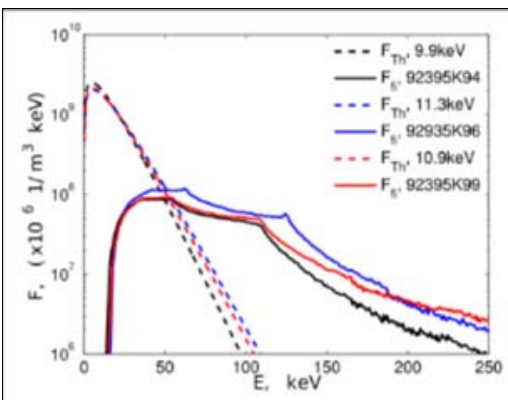

a)

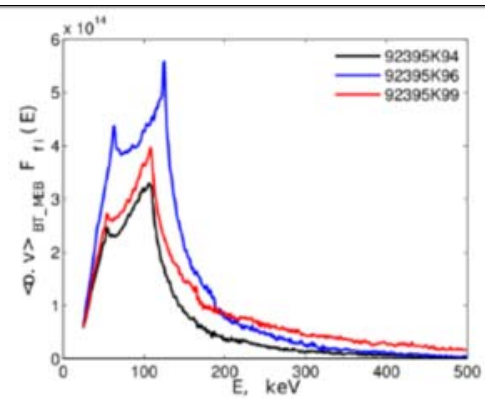

b)

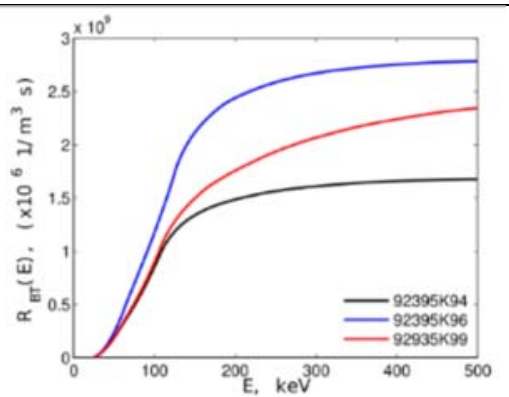

c)

Figure 14: Hybrid pulse \#92395, FI DF $\mathrm{F}_{\mathrm{fi}}$ (solid lines) and thermal Mawellian DF $\mathrm{F}_{\mathrm{Th}}$ (dashed lines) in a), the product $\langle\sigma . V\rangle_{\text {BT_MEB }} F_{f i} b$ ) and $B T$ cumulative reaction rate $R_{B T}(E)$ in $\left.c\right)$ near the magnetic axis, $R=3 \mathrm{~m}, \mathrm{Z}=0.23 \mathrm{~m}$ (position is also indicated by red dot in figure $13 \mathrm{~b}$ for $92395 \mathrm{~K} 94$ case). Reference case is in black, $4 \mathrm{MW}$ higher NB power is in blue and 4MW higher RF power shown by red lines.

Changes in fast ion distribution function in the core for hybrid pulse \#92395 after changing the auxiliary heating power by $4 \mathrm{MW}$ are shown in figures 14 and 15 . The product $\langle\sigma . V\rangle_{\text {BT_MEB }} F_{\text {fi }}$ and $B T$ cumulative reaction rate $\mathrm{R}_{\mathrm{BT}}(\mathrm{E})$ are shown as well.

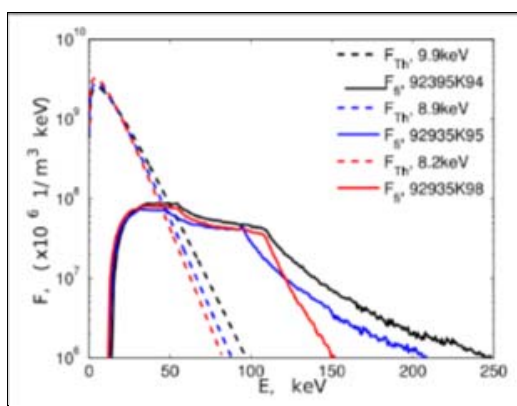

a)

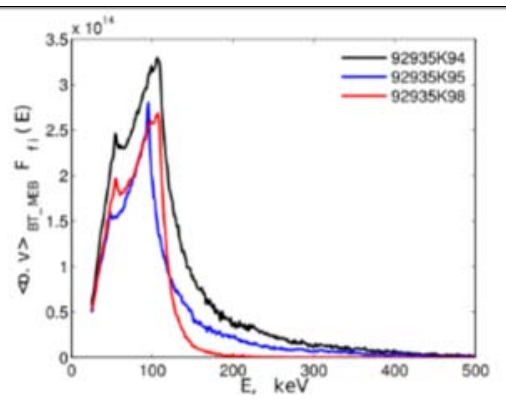

b)

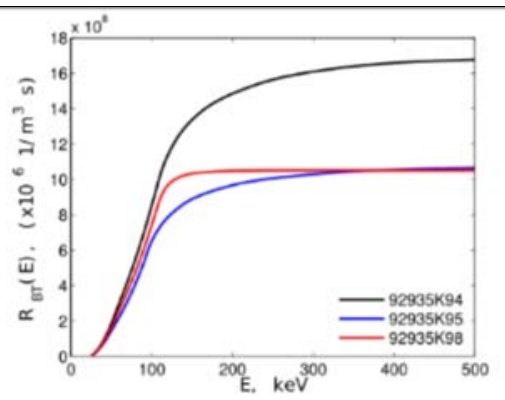

c)

Figure 15: same as the figure 14 but for the case of lowering the NB (blue) and RF (red) power by $4 \mathrm{MW}$.

Similar to the baseline case, RF power (red lines in figure 14 a) and 15 a)) affects to the highest extent the tail of the fast ion distribution function. By increasing the RF power from $1 \mathrm{MW}$ to $5 \mathrm{MW}$ and then to $9 \mathrm{MW}$ the contribution of energetic particles with $\mathrm{E}>120 \mathrm{keV}$ to the $\mathrm{BT}$ reactivity becomes dominant as seen from the red curves figure $14 \mathrm{~b}$ ) and $15 \mathrm{~b}$ ). At the highest RF power (case \#4 of table 2 and red line in figure $14 \mathrm{c}$ )) energetic particles with $E>110 \mathrm{keV}$ provide more than half, $54 \%$, of BT neutrons, while for the lowest RF power only about $16 \%$ of the integral of $\langle\sigma . v\rangle_{\text {BT }} F_{f i}$ comes from particles in the tail, case \#3 of table 2 and red line in figure $15 \mathrm{c}$ ).

NB power impacts mainly on the FI DF plateau, the higher energy end of which extends further with power as beam energy increases. The tail in the distribution function also changes but its slope remains relatively unchanged with $\mathrm{P}_{\mathrm{NB}}$, blue and black lines in figures $14 \mathrm{a}$ ) and 15 a). The contribution of the plateau to the BT reactivity slightly changes with NB power, about $62 \%$ for the 
highest $P_{\text {NB }}$ (case \#2 in table 2 with $30.8 \mathrm{MW}$ and blue line in figure $14 \mathrm{c}$ )) to about $56 \%$ for the lowest NB power (case \#1 in table 2 and blue line in figure $15 \mathrm{c}$ )).

It is obvious from figures 11 and 14 following the red curves that in the conditions of the best performing baseline and hybrid pulses RF power can generate an energetic tail in the fast ion distribution function to provide up to about half of the BT neutrons. In some cases, however, fusion performance enhancement due to synergistic effects can be much higher. For instance, in the three ion heating experiments performed recently at JET [51] very high energy tail in FI DF in this case is found to be responsible for more than $90 \%$ of generated DD neutrons.

\section{Conclusions}

Changes in auxiliary heating power, NB and RF, by 4MW against background of about 33MW will affect $D D$ fusion performance and neutron rates significantly in both scenarios studied here: baseline and hybrid. This will come through changes in bulk plasma parameters, $T_{e}$ and $T_{i}$, as well as in fast ion density and distribution function. Changes in ion temperature, $T_{i}$, will naturally affect thermal neutrons, while in addition to this changes to the fast ion distribution function will have an impact on BT neutrons. Thermal reaction rates, $R_{\text {Th, }}$, change within $12 \%$ to $60 \%$. Tables 1 and 2 show significant changes of averaged $B$ T reactivities, $\langle\sigma . v\rangle_{B T}$, and reaction rates, $R_{B T}$, more that about $20 \%$ in all cases, resulting in changes in BT neutrons, $\mathrm{N}_{\mathrm{BT}}$, between $30 \%$ and $60 \%$. The total neutron yields, $\mathrm{N}_{\text {TOT, }}$, vary within 25 to $52 \%$.

The impact of the RF and NB power on the BT reactivities $\langle<\sigma . v\rangle_{B T}>$ was found to be of similar order. From these observations one can conclude that in NB and RF plasma it is equally efficient for the DD BT reactions to act on the FI DF plateau by further increasing the NB power or to pull a high energy tail in the fast ion distribution function by applying higher RF power. Despite similarities in $<<\sigma . v>_{\mathrm{BT}}>$ variations with NB and RF power, it has been observed that NB power has greater impact than $R F$ regarding $\left\langle R_{B T}>\right.$ and $N_{B T}$ changes. This is attributed to the larger increase in $n_{f i}$ with $P_{N B}$ than with RF.

Investigating the pure impact of RF power by using the kinetic profiles from reference pulses but turning off the RF heating shows a moderate impact of the sheer synergistic effects on the fusion performance, normally a drop by about $10 \%$ in neutron rate is observed if RF power is removed. The supplementary effect accompanying application of RF power, i.e. changes in $T_{e}$ and $T_{i}$, play an important role in fusion performance. Examining the cumulative rates show that the RF tail in the modelled FI DF in the investigated high-performance pulses is not sufficiently large to provide significant enhancement in the high energy range on the fast ions.

Based on the simulations, one can conclude that both scenarios, baseline and hybrid, will equally benefit from synergistic effects. Both scenarios will benefit from adding $4 \mathrm{MW}$ of extra power to the reference pulses with NB power change having larger effect on neutron yield than RF. Dropping the RF power however will have a massive negative impact regarding fusion performance of both scenarios. An interesting observation is that similar changes in $T_{i}$ will inflict larger impact on thermal yield for baseline compared to hybrid. Also comparing the two scenarios it seems hybrid will compensate the smaller increase in thermal yield by larger increase in BT neutrons with power. 
Regarding planning future experiments with the goal of achieving maximum DD fusion performance, the simulations point out that for the baseline scenario an effort to increase the NB power will be more beneficial, while RF power should not be dropped below 5-6MW. Similar conclusions can be drawn for the hybrid pulses. Providing that changes in BT reactivities with NB and RF power are of the same order, clearly the conclusion that NB will have more beneficial impact on BT reaction rate is based on the fact that $n_{f i}$ increases more with $P_{N B}$ than with $P_{R F}$.

The scope of the study can be further extended in analysing the RF parameter space, e.g. minority scheme and concentrations, antenna phasing, etc. A challenging part of these studies will be to analyse DT mixture plasma. It will require better understanding of the isotope effect on the transport, confinement and pedestal physics scaling. This investigation can eventually provide the necessary predictive capabilities for the forthcoming DT campaign at JET.

\section{Acknowledgement}

This work has been carried out within the framework of the EUROfusion Consortium and has received funding from the Euratom research and training programme 2014-2018 under grant agreement No 633053. The views and opinions expressed herein do not necessarily reflect those of the European Commission.

\section{References}

[1] J-M. Noterdaeme et al., 17th IAEA Conf. on Fusion Energy, IAEA-CN-69/CDP/11, Yokohama, 1998

[2] A. M. Messiaen et al 1993 Plasma Phys. Control. Fusion 35 A15-34

[3] M. Mantsinen et al 2004 Nucl. Fusion 44, p.33

[4] D. van Eester et al 2009 Plasma Phys. Control. Fusion 51044007

[5] E. Lerche et al 2009, Plasma Phys. Control. Fusion 51, 044006

[6] M. Schneider et al 2006 Nucl. Fusion 56112022

[7] H. Henriksson et al 2006, Nucl. Fusion 46, p.244

[8] D. Gallart et al 2017 Europhysics Conference Abstracts vol. 40A (2017) P2.003

[9] M. Mantsinen et al European Journal of Physics 157 (2017) 03032

[10] D. Gallart et al European Journal of Physics 157 (2017) 03015

[11] D. Gallart, M. Mantsinen et al 2018, Nucl. Fusion 58, in press

[12] J. Jacquinot and JET Team 1998, Nucl. Fusion 38, p.1263

[13] G. A. Cottrell et al 1989 Plasma Phys. Control. Fusion 31, p. 1727

[14] L.-G. Eriksson, M. Mantsinen et al 1999, Nucl. Fusion 39, p.337

[15] M. Mantsinen et al 2001, Nucl. Fusion 41, p.1815

[16] G. A. Cottrell, F. G. Rimini and JET team 1999, Nucl. Fusion 39, p. 2025

[17] D. F. H. Start et al 1999, Nucl. Fusion 39, p. 321

[18] D. F. H. Start et al 1998, Phys. Rev. Lett. 80, 4681

[19] D. F. H. Start and JET team 1998, Plasma Phys. Control. Fusion 40, p. A87

[20] D. M. Meade and the TFTR team 1994, Journal of Fusion Energy, Vol. 13, p. 145

[21] R. V. Budny et al 1995, Nucl. Fusion 35, p.1497

[22] E. Joffrin and JET contributors 2018 27th IAEA Fusion Energy Conference, Ahmedabad, India

[23] A. Fasoli et al 2007, Nucl. Fusion 47, p. S264

[24] ITER Physics Expert Group on Energetic Particles Heating and Current Drive 1999, Nucl. Fusion 39, p. 2495

[25] J. Garcia, L. Garzotti, M. Nocente et al 2018 27th IAEA Fusion Energy Conference, Ahmedabad, India

[26] E. J. Doyle et al 2007, Nucl. Fusion 47, p. S18 
[27] L. Garzotti et al 2018, 27th IAEA Fusion Energy Conference, Ahmedabad, India

[28] M. Romanelli et al 2014, Plasma and Fusion Research 93403023

[29] M Erba et al 1997, Plasma Phys. Control. Fusion 39, p.261

[30] L-G. Eriksson et al 1993, Nucl. Fusion 33, p1037

[31] P. Stubberfield and M. Watkins, JET-DPA(06)/87 1987

[32] C. Challis 1989, Nucl. Fusion 29, p.563

[33] M. Mantsinen et al 1999, Plasma Phys. Control. Fusion 41, p. 843

[34] R.J. Hawryluk et al "An Empirical Approach to Tokamak Transport", in Physics of Plasmas Close to Thermonuclear Conditions, ed. by B. Coppi, et al., (CEC, Brussels, 1980), Vol. 1, pp. 19-46

[35] R. V. Budny, M. G. Bell, H. Biglari, et al.1992, Nucl Fusion 32, p.429.

[36] http://w3.pppl.gov/transp

[37] B. A. Grierson et al 2018, Fus. Sci. Techn., DOI: 10.1080/15361055.2017.1398585

[38] A. Pankin, D. McCune, R. Andre et al., "The Tokamak Monte Carlo Fast lon Module NUBEAM in the National Transport Code Collaboration Library", Computer Physics Communications Vol. 159, No. 3 (2004) 157-184.

[39] M. Brambilla 1999, Plasma Phys. Control. Fusion 41, p.1

[40] G.W. Hammett, "Fast Ion Studies of Ion Cyclotron Heating in the PLT Tokamak", Ph.D. Thesis (Princeton, 1986)

[41] C. Gowers et al 1995 Rev. Sci. Instrum. 66471

[42] E. de la Luna et al 2001 Rev. Sci. Instrum. 741414

[43] M. Gatu Johnson et al 2008 Nucl. Intrum. Methods A 591417

[44] C. Hellesen et al 2010, Plasma Phys. Control. Fusion 52085013

[45] J. Eriksson et al 2016 Comp. Phys. Comm. p.40

[46] M. Mikkelsen et al 1989, Nucl. Fusion 29, p.1113

[47] C. Bourdelle, J. Citrin et al 2016, Plasma Phys. Control. Fusion58 014036

[48] M. Goniche et al 2017, Plasma Phys. Control. Fusion 59, 055001

[49] J.G. Cordey et al 2003, Nucl. Fusion 43, p.670

[50] L. Frassinetti et al 2018, 45th EPS Conference on Plasma Physics, 2-6 July 2018, Prague, Czech Republic, P4.1027

[51] Ye. Kazakov et al 2015, AIP Conference Proceedings 1689, 030008

[52] F. J. Casson, H. Patten et al 2018 27th IAEA Fusion Energy Conference, Ahmedabad, India

[53] R. Budny, M. Gorelenkova, 57th Annual Meeting of the APS Division of Plasma Physics, Volume 60, Number 19, GP12.00127, 16-20 Nov 2015, Savannah, Georgia 